\title{
Sexually satiated male uses gustatory-to-neuropeptide integrative circuits to reduce time investment for mating
}

Woo Jae Kim ${ }^{1, *}$, Seung Gee Lee ${ }^{1}$, Anne-Christine Auge ${ }^{1}$, Lily Yeh $\mathrm{Jan}^{2}$, and Yuh Nung $\operatorname{Jan}^{2}$

${ }^{1}$ Department of Cellular and Molecular Medicine, University of Ottawa, Ottawa, Ontario K1H 8M5, Canada

${ }^{2}$ Howard Hughes Medical Institute, Departments of Physiology, Biochemistry and Biophysics, University of California San Francisco, San Francisco, California 94158, USA

*Correspondence: wkim@uottawa.ca

\section{ABSTRACT}

Males rely on a 'time investment strategy' to maximize reproductive success. Here we report a novel behavioral plasticity whereby male fruit flies exhibit a shortened mating duration when sexually satiated, which we named 'Shorter-Mating-Duration (SMD)'. SMD requires the sexually dimorphic Gr5a-positive neurons for detecting female body pheromones. The memory circuitry within the ellipsoid body (EB) and mushroom body (MB) brain regions is crucial for SMD, which depends on the circadian clock genes Clock and cycle, but not timeless or period. SMD also relies on signaling via the 
neuropeptide sNPF, but not PDF or NPF. Sexual experience modifies the neuronal activity of a subset of sNPF-positive neurons involved in neuropeptide signaling, which modulates SMD. Thus, our study delineates the molecular and cellular basis for SMD a plastic social behavior that serves as a model system to study how the brain switches the internal states between sexual drive and satiety. 


\section{INTRODUCTION}

From simple behaviors to sophisticated decisions, animals must make choices throughout their life in order to maximize their resources (Louâpre, van Alphen and Pierre, 2010). The reproductive success of a male animal is a function of the number of his sperm cells that are successful in fertilizing female eggs. Hence, sexual selection involves not just adaptive phenotypes for mating with many female partners (Parker, 1984). Males have a finite resource to spend on reproduction (Parker and Pizzari, 2010), which is limited by the number of ejaculates that can be delivered and the time required to restore depleted reserves (Dewsbury, 1982). Sperm can be costly to produce therefore males strategically allocate their investment into the ejaculate (Dowling and Simmons, 2012).

Besides ejaculate economics, time waste also can constitute a considerable selective disadvantage for males due to increased exposure to environmental hazards such as predators, or a lower fertilization rate, than their competitors. Regarding adaptation, time waste can be defined as where an individual spends longer than other animals to complete a given activity. Those individuals which waste time might expose themselves to the action of predators or various environmental hazards, then eventually fall into less competitive situations. In a species with a continuous life history. Moreover, time waste in males can result in direct disadvantages of the species by increasing the generation interval (Parker, 1974). 
In a competitive mating environment, time-wasting males will have a significantly lower fertilization rate than their rivals. For males, the balance between the time investment for feeding and searching for females is the critical factor to increase their reproductive fitness (Parker, 1970). In this regard, a 'time investment strategy (optimum allocation of time spent on given activities to achieve the maximum reproductive success)' is crucial for males.

In support of the male's time investment strategy, recent studies have revealed that $D$. melanogaster males vary greatly in their level of interest in females, providing evidence that males have also evolved mate selectivity behavior (Gowaty, Steinichen and Anderson, 2003). When mating opportunities are constrained, males show a preference for more fecund females, and in turn will benefit directly by increasing the number of offspring they produce (Byrne and Rice, 2006a). The stringent mating investment by Drosophila males might have evolved for the following reasons. First, sexual activity reduces the lifespan of males (Partridge and Farquhar, 1981) due to costs arising from vigorous courtship (Cordts and Partridge, 1996), the production of ejaculates (Lefevre and Jonsson, 1962), and possibly immunosuppression (McKean and Nunney, 2001). Second, repeated mating by males within a day depletes limiting components of the ejaculate (Demerec and Kaufman, 1941; Lefevre and Jonsson, 1962). Third, the quality of potential female mates is highly variable (Lefranc and Bundgaard, 2000). Thus, male flies' efforts for mating might vary in different contexts.

Behavioral plasticity is preferable when specific aspects of the environment (e.g., the 
intensity of socio-sexual encounters) are prone to rapid and unpredictable variation (Bretman, Gage and Chapman, 2011). Behavioral plasticity requires the formation of association between a proper behavioral output and given information, as the consequences of multiple interactions between evolutionarily programmed innate behaviors and cumulated learning experiences of the animal (Dissel, Angadi, Kirszenblat, Suzuki, Donlea, Klose, Koch, English, Winsky-Sommerer, van Swinderen and Shaw, 2015; Lupold, Manier, Ala-Honkola, Belote and Pitnick, 2011).

What are the general terms of behavioral plasticity and what is its meaning in the context of the fruit fly's sexual behavior? One example of plastic male behavior is 'Longer-Mating-Duration (LMD)', with increased investment via mating duration lengthening induced by exposure to rivals before mating (Bretman, Fricke and Chapman, 2009). In Drosophila, males respond to the presence of rivals by prolonging mating duration to guard the female and pass their genes. In previous studies, we examined the genetic network and neural circuits that regulate rival-induced longer mating duration (LMD). LMD can be induced solely via visual stimuli. LMD depends on the circadian clock genes timeless and period, but not Clock or cycle. LMD involves the memory circuit of the ellipsoid body (EB). Further, we identified a small subset of clock neurons in the male brain that regulates LMD via neuropeptide signaling (Kim, Jan and Jan, 2013).

Here we report a novel plastic behavior of male $D$. melanogaster for its selective investment in mating. Sexually satiated Drosophila males show this plastic behavior by 
limiting their investment in copulation time, namely 'Shorter-Mating-Duration (SMD)'. In addition to delineating the requisite circuitry by identifying the neurons for SMD, our study has uncovered the sensory stimuli, clock genes, sexual dimorphism, and neuropeptide signaling crucial for the SMD behavior, which is functionally and mechanistically distinct from the previously identified LMD pathway. 


\section{RESULTS}

\section{Sexually satiated males reduce investment in mating duration as compared to naïve males.}

To investigate how sexual experience affects the mating duration of male fruit flies, we introduced virgin females into group-reared males 1 day before the assay (this condition will be referred as 'experienced' henceforward), and compared their mating duration with group-reared males that never encountered sexual experience (this condition will be referred as 'naïve' henceforward) (Figure 1A). We found that the mating duration of wild-type naïve males such as Canton-S (CS), WT Berlin $\left(W T^{\text {Ber }}\right)$, and Oregon R (Ore $\left.{ }^{R}\right)$ is significantly longer than that of sexually experienced males (Figure 1B). To test whether the genetic background of male flies affect the SMD behavior, we performed mating duration assay with laboratory maintained $w^{1118}$ males. We found in our previous study that $w^{1118}$ males do not show the LMD behavior as a plastic responses of male fruit flies to the level of sperm competition, which results in significantly increased reproductive success in a competitive environment by extending mating duration (Bretman, Fricke and Chapman, 2009), probably because $w^{1118}$ males have defective vision (Kim et al., 2013). In contrast, we found the SMD behavior was normal in $w^{1118}$ mutant ( $w^{1118}$ in Figure $1 \mathrm{~B}$ ). We also found that socially isolated males show SMD behavior (Figure S1A). Thus, unlike LMD, SMD persists in either grouped or isolated rearing conditions. Therefore, we decided to perform all mating duration assays with a group-reared condition. 
We wondered whether the reduced mating duration of sexually experienced males may have been caused by the fatigue of repetitive sex. To test whether fatigue causes SMD behavior, we examined other behavioral repertories of naïve and experienced male flies, such as courtship index (a measure of the time a male engages in any defined recognizable courtship behaviors), courtship latency (time to courtship initiation), copulation latency (time to copulation/mating initiation), and locomotion (any of a variety of movements or methods that animals use to move from one place to another), and found no significant difference between experienced and naïve males (Figure S1B-G). Since several behavioral repertories of experienced males that should be affected by their fatigue were comparable to those of naïve males, we conclude that fatigue of repetitive female experiences is not a factor in causing SMD behavior.

Recently Zhang and colleagues reported that male flies show reproductive satiety when males are exposed to an excessive number of females. In their experimental paradigm, the percentage of time males spend in mating behaviors gradually declines to $\sim 10 \%$ over $4 \mathrm{~h}$ (Zhang, Rogulja and Crickmore, 2016). This finding suggests that males do not invest much time for mating behaviors when they are satiated with females after $4 \mathrm{~h}$ of mating experiences. To test whether the SMD behavior is different from reproductive satiety, we designed a series of experiments by varying the time of male exposure to females. We found that males shortened their mating duration when their exposure to females lasted for $12 \mathrm{~h}$ rather than $6 \mathrm{~h}$ or $2 \mathrm{~h}$, suggesting that SMD requires chronic exposure to females for longer than $6 \mathrm{~h}$ (Figure 1C). This suggests that, whereas $4 \mathrm{~h}$ of 
mating experience can induce reproductive satiety and reduce the mating drive, it is not sufficient to induce SMD behavior.

One of the crucial features of behavioral plasticity is the reversibility, which allows animals to adapt to fast changing environment. To determine whether SMD is a reversible behavior, we isolated males from females after $24 \mathrm{~h}$ of sexual experience then performed mating duration assay. We found that separating experienced males from females for $24 \mathrm{~h}$ was sufficient to restore the MD to the level of naïve males (Figure 1D). All these data suggest that SMD is plastic; it can change over time and may vary with the context, and is dependent on sexual experience with females.

Males should be especially prudent in the allocation of their limited resources such as the amount of sperms, which determines the reproductive success of male fruit fly (Lupold, Manier, Ala-Honkola, Belote and Pitnick, 2011). Given that sperm depleted males prefer large females as partners in courtship and copulation (Byrne and Rice, 2006b), we hypothesized that sperm depletion of experienced males may affect SMD behavior. To test the effect of sperm depletion on mating duration, we designed a series of experiments by varying the number of virgin females presented to a male, by up to a factor of 10. Because $5 \mathrm{~h}$ exposure with 4 females (Byrne and Rice, 2006b) or four consecutive copulation with females (Demerec and Kaufmann, 1941; Lefevre and Jonsson, 1962) depletes the majority of sperms, we decided to introduce 10 females to a male for a certain amount of time in order to deplete most of his sperm. We found that the mating duration of a male exposed to 10 females for $2 h, 4 h$, or $8 \mathrm{~h}$ is comparable 
to that of control males (Figure $\mathrm{S} 1 \mathrm{H}$ ). To further confirm the lack of effect of sperm depletion on SMD behavior, we tested the son-of-tudor males that lack germ cells and therefore are spermless (Xue and Noll, 2000). Spermless son-of-tudor males show the intact SMD behavior suggesting that the absence of germ cells do not affect SMD behavior (Figure S1I). Consistent with our findings, Crickmore and colleagues have shown that the mating time of male fruit fly is not determined by the volume or rate of transfer of reproductive fluids (Crickmore and Vosshall, 2013). All these data suggest that sperm depletion of male does not cause SMD behavior.

To look for the physiological cues from females that produce SMD behavior, we first introduced various types of females as sexual partners to condition males so they would become "experienced", before mating duration assay. We found that mated females and D. pseudoobscura virgin females cannot induce SMD behavior (fe $e^{\text {mated }}$ and $f e^{D . p s e}$ in Figure 1E). Mated females are known to display the post-mating responses (PMR) via sex peptide (SP)/sex peptide receptor (SPR) signaling and reject males (Yapici, Kim, Ribeiro and Dickson, 2008). It is also known, and confirmed in our experiments, that male $D$. melanogaster cannot mate with $D$. pseudoobscura females (data not shown). Next, we asked whether mating experiences with $D$. melanogaster females are crucial to producing SMD. We first tested SPR deficiency mutant females, $D f(1)^{\text {exel6234 }}$, that lack the SPR protein and remain receptive to males, exhibiting virgin-like behaviors after mating (Hasemeyer, Yapici, Heberlein and Dickson, 2009; Rezaval, Pavlou, Dornan, Chan, Kravitz and Goodwin, 2012; Yang, Rumpf, Xiang, Gordon, Song, Jan and Jan, 2009; Yapici, Kim, Ribeiro and Dickson, 2008). Males experienced with the sex-peptide 
receptor (SPR) mutant females exhibited the normal extent of SMD $\left(D f(1)^{\text {exe/6234 }}\right.$ in Figure 1E). We then asked how SMD behavior may be affected if males were conditioned via exposure to virgin females behaving like mated ones. We found that SMD could not be induced by virgin females with expression of the membrane-bound form of male sex-peptide in fruitless-positive neurons, which behave like mated females that are not receptive to mating (Hasemeyer, Yapici, Heberlein and Dickson, 2009; Yang, Rumpf, Xiang, Gordon, Song, Jan and Jan, 2009; Yapici, Kim, Ribeiro and Dickson, 2008) (fru ${ }^{U A S-m S P}$ in Figure 1E). These results suggest that the mating experiences are necessary to induce SMD behavior.

We next asked what kinds of physiological cues are provided by females to induce SMD behavior of males. Oenocytes are secretory cells that are the major sites synthesizing cuticular hydrocarbons including pheromones, which is critical to insect communication (Makki, Cinnamon and Gould, 2014). To test whether the cues produced from the oenocytes are essential to induce SMD, we produced pheromone-free females by ablating female oenocytes (oeno(-)). To test whether the female form but not the male form of cuticular hydrocarbons is required to generate SMD behavior, we generated females expressing a male-odor by masculinization of female oenocytes (oe $\left.{ }^{\text {tra-RNAi }}\right)$. We confirmed that these females show normal mating behavior with wild type males (data not shown). Males experienced with these females did not show SMD behavior (oeno(-) and $o e^{\text {tra-RNAi }}$ in Figure 1E), suggesting that female-specific pheromones produced in oenocytes are important cues for inducing SMD. If an oenocyte-masculinized female served as the mating duration assay partner rather than females providing mating 
experiences to males in the experienced group, the SMD remained normal (fe ${ }^{\text {assayed }}$ in Figure 1E). This data suggests that male's decision on mating duration heavily depends on the previous mating experiences, not the current one. All these findings indicate that the mating experience and the $D$. melanogaster female-specific odors produced by oenocytes are both required to induce SMD behavior.

\section{Contact chemoreception is critical for inducing SMD behavior}

Male flies can detect females with various sensory modalities. In the early steps of courtship rituals, mainly visual, vibratory and olfactory signals allow males to find females and to orient towards females. In the next steps, males contact females by tapping with their forelegs to detect non-volatile hydrocarbon pheromones on the female cuticle via the gustatory system (Fernandez and Kravitz, 2013). To identify the sensory modalities that modulate SMD behavior, we tested the following mutants with defects on each sensory modality as described before (Kim, Jan and Jan, 2012, 2013). To test the visual contribution to SMD behavior without genetic intervention, we first performed an MD assay with animals reared in constant darkness for 5 days. SMD behavior was intact under constant dark conditions (dark in Figure 2A). Blind males with photoreceptors removed via GMR-hid also showed SMD (GMR ${ }^{\text {Hid }}$ in Figure 2A). SMD was also normal in mutants with impaired vision, such as nina $E^{17}$ without rhodopsins in R1-6 photoreceptors (ninaE ${ }^{17}$ in Figure 2A) (Cook, Pichaud, Sonneville, Papatsenko and Desplan, 2003). Indeed, visually defective $w^{1118}$ mutant also displayed normal SMD behavior (Figure 1B). All these data suggest that vision is not important for SMD 
behavior.

To test the possible involvement of the olfactory pathway, we performed mating duration assay with olfactory mutants $\left(\mathrm{Orco}^{1} / \mathrm{Orco}^{2}\right)$ with disrupted behavioral and electrophysiological responses to many odorants (Louis, Huber, Benton, Sakmar and Vosshall, 2008). Interestingly, the $\mathrm{Orco}^{1} / \mathrm{Orco}^{2}$ trans-heterozygote mutant displayed normal SMD behavior (Orco ${ }^{1} / \mathrm{Orco}^{2}$ in Figure 2A), suggesting that the olfactory pathway is not crucial for SMD induction. We then asked whether the gustatory pathway is important for SMD behavior. Gustatory mutants $\left(G u s t D^{x 6}\right)$ with aberrant responses to sugar and $\mathrm{NaCl}$ (Rodrigues, Sathe, Pinto, Balakrishnan and Siddiqi, 1991) did not exhibit SMD behavior (GustD $D^{x 6}$ in Figure $2 \mathrm{~A}$ ), suggesting that the gustatory pathway is a critical sensory modality to induce SMD behavior. We also tested the auditory mutant $\operatorname{iav}^{1}$ (Bretman, Westmancoat, Gage and Chapman, 2011) and found that they also did not exhibit SMD (iav ${ }^{1}$ in Figure 2A). In summary, we found evidence for the involvement of gustatory and auditory pathways in generating SMD, although we cannot rule out the possible involvement of other sensory stimuli.

Flies touch one another upon their encounter. Taste and touch signals are conveyed to the brain by sensory neurons in the legs and mouthparts (Billeter and Levine, 2013). Male flies can detect pheromones by pheromone-sensitive cells that are incorporated into the chemosensory systems of taste and smell. We decided to focus on the taste system since we found that olfactory stimuli are not critical to induce SMD behavior (Figure 2). So we next asked whether we can identify the particular types of male taste 
neurons that are essential to detect female cues in eliciting SMD behavior. As reported previously (Wang, Singhvi, Kong and Scott, 2004), Gr5a- and Gr66a-positive neurons in the fly gustatory system correspond to two non-overlapping neuronal populations. Gr5apositive cells mediate sweet-taste detection, whereas Gr66a-positive cells mediate bitter-taste recognition. We found that male flies with ablated Gr5a-positive neurons cannot elicit SMD behavior while male flies lacking Gr66a-positive neurons show normal SMD (Figure 2B), suggesting that Gr5a-positive neuronal populations are required for SMD. SMD was also impaired by by expressing Kir2.1 potassium channel in Gr5apositive neurons in an adult-specific manner using tub-GAL80 ${ }^{t s}$ (Figure S2A). To identify those neurons that express Gr5a or Gr66a, we characterized the expression pattern driven by the Gr5a-GAL4 or Gr6a-Gal4 driver using the reporter UAS-mCD8GFP (a cell membrane marker) along with UAS-RedStinger (a nuclear marker). We confirmed that these two neuronal populations have non-overlapping axonal projections to the suboesophageal ganglion (SOG) region in both males and females (Figure $2 \mathrm{C}$ ) (Wang, Singhvi, Kong and Scott, 2004). All these results confirm that the Gr5a taste neurons are essential for generating SMD behavior.

Pheromones and the mechanisms for their detection have evolved to transmit biologically relevant information from one member of a species to another (MucignatCaretta, 2014). Whereas the olfactory system is important for volatile pheromone communications, insect gustatory neurons present on the tarsi (legs) are also required for courtship behavior, and are selective for the detection of male or female cuticle lipids, to mediate 'contact chemoreception' (Mucignat-Caretta, 2014). LUSH is an 
odorant-binding protein (OBP) expressed in the antenna, and is also found in a subset of tarsal chemoreceptors on the forelegs (Kim, Repp and Smith, 1998). We asked whether the expression of LUSH in Gr5a-positive neurons is responsible for pheromone signaling of SMD induction. Knocking down the expression of LUSH in Gr5a-positive neurons diminished the SMD behavior, whereas knocking down LUSH expression in Gr66a-positive neurons had no effect on SMD (Figure 2D), suggesting that LUSH expression in Gr5a-positive neurons are crucial for detecting female pheromones for producing SMD behavior.

Snmp1 is in the CD36 related protein family and functions as an important player for the rapid kinetics of the pheromone response in insects (Li, Ni, Huang and Montell, 2014). We found that various genotypes of snmp1 mutants did not show SMD behavior (Figure S2B), suggesting that expression of Snmp1 protein is necessary for SMD behavior. Snmp1 is prominently expressed in olfactory sensory neurons in the antenna, and is also expressed in chemosensory organs on the proboscis (Benton, Vannice and Vosshall, 2007). We found that expression of Snmp1 in the snmp1 mutant background via the Gr5a-GAL4 driver could rescue SMD behavior (Figure 2E), suggesting that expression of Snmp1 protein only in Gr5a-positive neurons is sufficient to induce SMD behavior. Taken together, these data suggest that contact chemoreception mediated by the pheromone sensing proteins LUSH and Snmp1 in Gr5a-positive gustatory neurons is critical for triggering SMD behavior.

\section{Male-specific Gr5a-positive neurons are required for SMD}


Mating duration of $D$. melanogaster is solely determined by male and is associated with significant fitness benefits (Bretman, Westmancoat and Chapman, 2013). We previously showed that a small number of male-specific neurons control the rival-induced extended mating duration, LMD behavior (Kim, Jan and Jan, 2013). Since SMD behavior is also associated with modulation of mating duration, we hypothesized that male-specific and sexually dimorphic neural circuits modulate this behavior. It is well known that sexual dimorphism of sensory structure and function generates neuronal circuitries important for gender-specific behaviors (Zarkower, 2001). In Drosophila, fruitless (fru) is an essential neural sex-determinant responsible for male-specific behaviors (Ryner, Goodwin, Castrillon, Anand, Villella, Baker, Hall, Taylor and Wasserman, 1996). To determine whether sexually dimorphic sensory neurons are involved in SMD, we used intersectional methods to genetically dissect $\sim 1500$ fru neurons into smaller subsets. We used a combination of the $f r u^{F L P}$ allele that drives FLP-mediated recombination specifically in fru neurons with UAS[stop]X (X could be various reporters or effector transgenes) to express a UAS transgene in those cells that are not only labeled by the GAL4 driver but are also fru-positive, due to FLP-mediated excision of the stop cassette ([stop]) (Yu, Kanai, Demir, Jefferis and Dickson, 2010a).

We first asked if there are fru-positive and sexually dimorphic sensory neurons among Gr5a-positive cells. When we used Gr5a-GAL4 to identify sexually dimorphic Gr5apositive cells, we found a restricted numbers of FRU-positive and Gr5a-positive neurons 
in the male forelegs and proboscis (compare GFP to Stinger in top panels of Figure 3A). In contrast, we could not find any FRU-positive and Gr5a-positive neurons in female foreleg and proboscis (middle panels in Figure 3A), suggesting that these FRU-positive and Gr5a-positive sensory neurons are sexually dimorphic. We failed to label FRUpositive neurons in male forelegs and proboscis using Gr66a-GAL4 driver (bottom panels in Figure 3A), suggesting that there are no FRU-positive and Gr66a-positive sensory neurons in male's forelegs and proboscis. Next we asked whether these malespecific neurons project their axons into the SOG (suboesophageal ganglion) region receiving gustatory inputs. We confirmed the axonal projections of these male-specific Gr5a-positive neurons to the SOG region with the membrane marker UAS-mCD8GFP (Figure 3B) and the presynaptic marker UAS-nsybGFP (Figure 3C). We could not find any GFP signals with this manipulation within the female brain (Figure S3A). The same genetic manipulation to label FRU-positive and Gr5a-positive neurons with the dendritic marker UAS-DscamGFP failed to label Gr5a-positive GFP signals within the SOG region (Figure S3B), suggesting that the GFP fluorescence shown in Figure 3B corresponds to presynaptic axon terminals, instead of dendrites. All these data suggest that sexually dimorphic Gr5a-positive and FRU-positive neurons in male forelegs and proboscis project their axons into the SOG region of the male brain.

We next analyzed the function of these male-specific neurons on SMD behavior. We first confirmed that inhibiting synaptic transmission by TNT expression via Gr5a-GAL4 but not Gr66a-GAL4 specifically eliminated SMD behavior (Figure S3C). To test whether the small subset of FRU-positive cells are involved in SMD, we expressed tetanus toxin 
light chain (UAS[stop]TNT $T_{\text {active }}$ ) with Gr5a- or Gr66a-GAL4 drivers along with fru ${ }^{F L P}$ to inhibit synaptic transmission in sexually dimorphic subsets of FRU-positive cells. Surprisingly (why is this surprising?), we found that SMD was abolished when UAS-TNT was expressed only in male-specific Gr5a-positive neurons (Figure 3D). As a control, we found that SMD was unaffected when we used each of these GAL4 drivers in combination with UAS[stop]TNT $T_{\text {inactive }}$ to express an inactive form of tetanus toxin light chain (Figure 3E). These data suggest that inhibition of synaptic transmission only in the male-specific Gr5a-positive neuronal population could specifically block the sensory inputs that induce SMD behavior.

Next, we tested for the effect of transforming these sensory neurons into the female form. It is well known that systemic expression of the female form of tra cDNA (UAS$\operatorname{tra}^{F}$ ) in a male brain during development elicits female characteristics (Belote and Baker, 1987). Interestingly, we found that SMD was eliminated by the feminization of

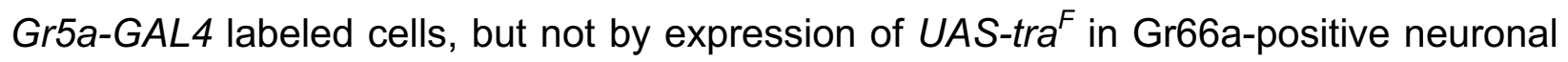
subsets (Figure 3F), suggesting that feminization of Gr5a-positive neurons nullify the male-specific sensory function of those cells to detect female cuticular pheromones and induce SMD behavior. All these data suggest that SMD requires the male-specific role of a subset of Gr5a-positive neurons. Together, these results suggest that the odorant binding proteins LUSH and coregulatory protein SNMP1 in male-specific Gr5a-positive neurons are crucial to send satiety signals to the central brain to produce SMD behavior. 


\section{The circadian clock genes Clock and cycle are involved in SMD}

One of the most crucial features of the central brain is to integrate temporal information with precise physiological responses. Evolution has favored different scales of biological timing from the $24 \mathrm{~h}$ circadian system to the seconds-to-minutes range of interval timing, and to the millisecond timing that is crucial for speech generation, motor control, sound localization, and consciousness of human being (Golombek, Bussi and Agostino, 2014). Among those different scales of biological timing, 'interval timing' is associated with essential behaviors such as foraging, learning, and decision making (Tucci, 2011). In human, it is the function of interval timing that allows us to experience the passage of real time subjectively. A human being can integrate action sequences, thoughts and behavior when interval timing functions properly. Our ability to detect emerging trends and to anticipate future outcomes also largely depends on the interval timing function of our brain (Block and Grondin, 2014; Buhusi and Meck, 2005). We suggested that the rival-induced extended mating, so called LMD behavior, could be a model for studying interval timing in Drosophila melanogaster (Kim, Jan and Jan, 2012, 2013). Also, here we suggest that SMD behavior can be an additional model system to investigate the neural basis of interval timing in the brain since both LMD and SMD behaviors involve decision-making procedures that take minutes.

The circadian clock is the most well understood features among biological timing in molecular terms (Hall, 2005). It constitutes of well-defined transcription/translationbased negative feedback loops that are controlled by core clock genes such as 
timeless, period, Clock, and cycle (Allada and Chung, 2010). In contrast to the circadian clock, the molecular components and the neural mechanisms for interval timing is not well understood. It has been suggested that the circadian clock may influence the interval timer, however, the molecular mechanisms is not known (Buhusi and Meck, 2005).

In Drosophila, the Clock $(C / k) / c y c l e(c y c)$ dimer activates the transcription of period (per), timeless (tim), vrille (vri), PAR domain protein 1 (Pdp1) and clockwork orange (cwo) genes, which in turn feedback to inhibit CLK-activated transcription or regulate Clk transcription (Zheng and Sehgal, 2008). In addition to regulating circadian rhythm of the animal, clock genes regulate the timing of non-circadian phenomena, such as the frequency of the male courtship song, developmental time, sleep length, cocaine sensitization, and giant fiber habituation (Hall, 2005). Our previous study revealed that tim and per are specifically involved in LMD (Kim, Jan and Jan, 2012).

To elucidate the relationship between circadian clock and interval timing, we tested the SMD behavior of core circadian clock gene mutants. We found that Clk and cyc mutant males did not show SMD whereas per and tim mutant males showed SMD (Figure 4A). Next, we asked whether the function of clock genes in the nervous system is sufficient to produce SMD behavior. Expression of CYC in cyc mutants with the pan-neuronal GAL4 driver could rescue SMD behavior (Figure 4B), suggesting that neuronal expression of CYC protein is sufficient to elicit SMD behavior. Pigment-dispersing factor (PDF) expressing clock neurons are known to be the core pacemaker neurons in the fly 
brain (Kim, Jan and Jan, 2013). To test whether the function of the cyc gene in PDFpositive neurons are essential to elicit SMD behavior, we used the pan-neuronal GAL4 driver combined with pdf-GAL80 to block the function of GAL4 specifically in PDFpositive cells. Interestingly, SMD defects in cyc mutants can be rescued with the introduction of CYC protein to neurons other than the PDF-expressing clock neurons (Figure 4B), suggesting that the function of CYC in PDF-expressing cells is dispensable for SMD behavior.

Next, we asked whether the function of clock genes on SMD behavior is adult-specific. Similar to previous findings that transgenic rescue of cyc mutants behavioral arrhythmia in adults depends on developmental cyc expression during metamorphosis (Goda, Mirowska, Currie, Kim, Rao, Bonilla and Wijnen, 2011), SMD could not be rescued by introducing CYC protein in an adult-specific manner indicating that the developmental function of CYC is required to elicit SMD behavior (Figure 4B). These data suggest that the developmental function of CLK/CYC in a subset of the neuronal population is required to induce SMD.

We then looked into the CLK/CYC function in SMD behavior. To identify the neuronal populations mediating the CLK/CYC function to elicit SMD, we screened for GAL4 drivers that eliminate SMD behavior when combined with Clk-RNAi or cyc-RNAi. Knocking down expression of CLK or CYC in the majority of clock neurons eliminated SMD (tim-GAL4 in Figure 4C and 4D). Expression of Clk-RNAi or cyc-RNAi in PDFexpressing lateral clock cells did not affect SMD (pdf-GAL4 in Figure 4C and 4D). 
Removal of CLK or CYC from CRY-positive clock neurons eliminated SMD (cry-GAL4 in Figure 4C and 4D), indicating that CLK/CYC expression in CRY-positive but PDFnegative cells is required for SMD, since CRY-positive neurons include all the PDFpositive cells in clock neurons. To confirm that PDF-positive neurons are dispensable for SMD behavior, we expressed UAS-Kir2.1 in PDF-positive neurons in an adult specific manner to specifically inhibit the neuronal activity of those cells. We found that inactivation of PDF neurons did not affect SMD ( $p d f$ in Figure S4A). Taken together, these data suggest that CLK/CYC function in a subset of PDF-negative clock neurons is required to process sexual satiety information by female experience to produce SMD behavior.

\section{SMD requires memory circuitry residing in the mushroom and ellipsoid bodies}

Males compete to find mates in a social environment (Wong and Candolin, 2005). In this circumstance, the ability of learning and memory can significantly increase the behavioral benefits of reproductive success of a male (Verzijden, ten Cate, Servedio, Kozak, Boughman and Svensson, 2012). In Drosophila, well-known behavioral paradigms such as "courtship conditioning" or "conditioned courtship suppression" have been widely used to study the theory of learning and the mechanisms of memory formation at the cellular and molecular level (Siegel and Hall, 1979). In most cases, the courtship conditioning paradigm has investigated the short-term memory (STM) lasting less than an hour. Long-term memory (LTM) forms in the fly brain when animals 
confront repetitive trials and a resting period, which lasts up to $24 \mathrm{~h}$ (Griffith and Ejima, 2009). Besides STM and LTM, mid-term memory (MTM) lasts from 1 to 3 hours. Moreover, two forms of long-term memory are distinguishable by training procedures, namely anesthesia-resistant memory (ARM) and long-term memory (LTM) (Heisenberg, 2003).

Sexual experiences can enhance the subsequent mating success through social learning and memory. Males can have a mating advantage over inexperienced males when they previously experienced mating. In this behavioral paradigm, courtship experience alone is not sufficient to provide a competitive advantage to the male. The copulation experiences play a significant role to reinforce the learning of males to perform more efficiently (Saleem, Ruggles, Abbott and Carney, 2014). Moreover, it has been reported that prior sexual experiences inhibit aggression between males. In this behavioral paradigm, $10 \mathrm{~h}$ or more experiences with females drastically suppress a male's aggression against rival male. (Yuan, Song, Yang, Jan and Jan, 2014). Moreover, we previously reported that previous experience of rival-enriched environment is stored as long-term memory in ellipsoid body neurons through the function of memory genes, rutabaga (rut) and amnesiac (amn) (Kim, Jan and Jan, 2012).

Hence, we asked whether SMD requires memory circuits. The memory trace of sexual experiences for generating SMD behavior disappears between $12 \mathrm{~h}$ and $24 \mathrm{~h}$ (Figure S4B), suggesting that SMD requires some long-term memory trace. Next, we tested 
whether SMD was altered in classical learning and memory mutants (dnc, rut, amn, and orb2). Interestingly, SMD was impaired only in orb2 mutants, but not in dnc, amn, or rut mutants (Figure 4E). However, orb2 mutants showed normal LMD behavior (Figure $\mathrm{S4C)}$. Thus orb2 function is important for the memory formation involved in SMD. Drosophila Orb2 belongs to the CPEB2 subfamily, which functions to stimulate mRNA translation. In courtship conditioning, Orb2 is a crucial component to form a long-term memory (Keleman, Kruttner, Alenius and Dickson, 2007).

Genetic intervention has provided strong evidence that the mushroom body (MB) regions act as the seat of memory for odors (Heisenberg, 2003). In contrast, visual pattern memory in $D$. melanogaster is linked to the central complex, which includes the ellipsoid body (EB) and fan-shape body (FB) (Liu, Seiler, Wen, Zars, Ito, Wolf, Heisenberg and Liu, 2006; Pan, Zhou, Guo, Gong, Gong and Liu, 2009). To test which brain region is important for memory processing in SMD behavior, we used several GAL4 lines that drive expression of UAS-Kir2.1 in $\mathrm{EB}, \mathrm{MB}$, or $\mathrm{FB}$ brain regions. Expression of UAS-Kir2.1 in the FB via 14-94-GAL4 had no effect on SMD (14-94 in Figure S4A), whereas expressing UAS-Kir2.1 in MB or EB regions abolished SMD (ok107 and c547 in Figure S4A), suggesting that EB and MB are the brain regions to generate memory for SMD behavior.

Having found a requirement of the MB and EB in producing SMD behavior, we performed GAL4-mediated RNAi knockdown of orb2 to identify the brain region where orb2 functions to process memory involved in SMD behavior. Knocking down orb2 
expression in the MB disrupted SMD (ok107 in Figure 4F), however, knocking down orb2 in the EB or FB had no effect on SMD (c547 and 14-94 in Figure 4F). These data suggest that the neural activity of $M B$ and $E B$ regions are both required for SMD, and orb2 expression in MB is likely required to process the memory required for SMD.

\section{Neuropeptide sNPF is crucial component of SMD behavior}

Neuropeptides regulate a wide range of animal behaviors. We found previously that LMD requires the functions of PDF and NPF but not SNPF in a subset of clock neurons (Kim, Jan and Jan, 2013). Since both LMD and SMD behaviors are related to modulated interval timing-based decision making, we tested whether the above neuropeptides expressed in clock neurons are involved with SMD behavior, by performing mating duration assays with mutants lacking a neuropeptide or its receptor. Since no npf mutant alleles are available (Nitabach and Taghert, 2008), we tested npfR1 mutants (Burke, Huetteroth, Owald, Perisse, Krashes, Das, Gohl, Silies, Certel and Waddell, 2012; Krashes, DasGupta, Vreede, White, Armstrong and Waddell, 2009). Among the mutants tested, males of mutant for npfR1 or pdf but not sNPF (Lee, Kwon, Lee, Kwon, Min, Jung, Kim, You, Tatar and Yu, 2008b) displayed SMD behavior (Figure $5 \mathrm{~A}$ and Figure S5A), indicating that SMD behavior only requires the function of neuropeptide sNPF, but not PDF or NPF.

Insect neuropeptides are expressed in discrete stereotypic neuronal populations in the central nervous system. Short neuropeptide F (sNPF) is the Drosophila homologue of 
the mammalian neuropeptide $\mathrm{Y}$ (NPY), which controls food consumption. sNPF is expressed in the nervous system and regulates food intake and body size (Lee et al., 2008b). sNPF is widely expressed in a variety of neurons in the fly brain including Kenyon cells of mushroom bodies. There are several thousands of diverse types of neurons in the adult fly brain expressing snpf transcript and sNPF peptide. It is known that the most of these neurons are inherent interneurons of mushroom bodies. Also, sNPF is expressed in many interneurons of the CNS, olfactory receptor neurons (ORNs) located in antennae, and the subpopulation of neurosecretory cells innervating the corpora cardiac and aorta (Nassel, Enell, Santos, Wegener and Johard, 2008). Even though the names of the two peptide genes (NPF and sNPF) are similar, previous reports showed that they play roles in two functionally distinct signaling pathways (Johard, Enell, Gustafsson, Trifilieff, Veenstra and Nassel, 2008; Lee, Kwon, Lee, Kwon, Min, Jung, Kim, You, Tatar and Yu, 2008a; Lee, You, Choo, Han and Yu, 2004; Wu, Wen, Lee, Park, Cai and Shen, 2003). These reports are consistent with our result. Our data suggest that the functions of these two neuropeptides do not overlap since sNPF but not NPF is involved with SMD behavior (Figure 5A), whereas NPF but not sNPF is involved with LMD (Kim, Jan and Jan, 2013).

We next wanted to identify the neurons that express SNPF to mediate SMD behavior. We used a previously verified UAS-sNPF-RNAi line (Lee, Kwon, Lee, Kwon, Min, Jung, Kim, You, Tatar and Yu, 2008b) in combination with various GAL4 drivers to knock down sNPF expression in discrete populations of GAL4 expressing cells. The brain regions labeled by the GAL4 drivers used in this study have been identified previously (Kim, Jan 
and Jan, 2012, 2013), as diagramed in Figure S5B. We found that SMD was abolished by expression of sNPF-RNAi in all neuronal population (via pan-neuronal elav-GAL4 driver, elav in Figure 5B) but not in glial cells (via repo-GAL4, repo in Figure 5B). We then tested the function of sNPF expression in sNPF-expressing cells and in cells expressing its receptor sNPFR1 using previously verified sNPF-GAL4 and sNPFR1GAL4 strains (Lee, Kwon, Lee, Kwon, Min, Jung, Kim, You, Tatar and Yu, 2008a). Knock down of sNPF using sNPF- or sNPFR1-GAL4 abolished SMD (sNPF and sNPFR1 in Figure 5B), indicating that sNPF-expression in a subset of cells that also express its receptor are important to induce SMD.

As described above, the circadian clock genes Clk and cyc are intimately involved with SMD behavior (Figure 4). Therefore, we tested GAL4 drivers that label different populations of clock neurons to identify the subset of clock neurons required for sNPF signaling. Interestingly, SMD remained intact with expression of sNPF-RNAi in PDFexpressing neurons ( $p d f$ in Figure $5 \mathrm{~B}$ ), but was abolished by expression of sNPF-RNAi in CRY-positive cells, which include most of the lateral neurons and a small subset of dorsal neurons (cry in Figure 5B). Thus, sNPF expression in neurons that express CRY but not PDF is required for SMD behavior. Expression of $s N P F-R N A i$ in a subset of dorsal neurons (C/k4.1M in Figure 5B) eliminated SMD, indicating that SNPF signaling in a subset of CLK-positive neurons is required for SMD.

We showed that SMD behavior requires memory circuit located in mushroom bodies (MB) and ellipsoid bodies (EB) (Figure S4A). Therefore, we next asked if sNPF 
signaling is critical to memory formation and processing for SMD behavior. We reasoned that knock down of sNPF signaling in mushroom bodies or ellipsoid bodies would disrupt SMD behavior if SNPF signaling plays a pivotal role in memory formation/processing. To characterize the relationships between sNPF signaling and memory circuits in SMD, we used different GAL4 drivers to express sNPF-RNAi in ellipsoid bodies (c547-GAL4), mushroom bodies (MB247- and ok107-GAL4), and fanshape bodies (FB) (14-94-GAL4). Whereas the expression of sNPF in EB neurons is required for SMD (c547 in Figure 5B), sNPF signaling in FB is dispensable for SMD (1494 in Figure 5B). When sNPF expression is reduced in MB via ok107-GAL4, SMD was eliminated, however knocking down sNPF expression via MB247-GAL4, which labels more restricted regions of the MB, did not affect SMD behavior (ok107 and MB247 in Figure 5B), indicating that sNPF signaling in MB neurons labeled by ok107-GAL4 but not MB247-GAL4 is crucial for SMD behavior (Jenett, Schindelin and Heisenberg, 2006).

We next asked what happens if an excessive amount of sNPF exists in a subpopulation of neurons that normally expresses sNPF or its receptor sNPFR1. Overexpression of UAS-sNPF via sNPF-GAL4 or sNPFR1-GAL4 had no effect on SMD behavior (Figure $\mathrm{S5C}$ ), indicating that increasing the amount of this neuropeptide in SNPF-positive or sNPFR1-positive neurons has no detrimental effect on SMD behavior.

Previous studies have shown that SNPF and its receptor sNPFR1 modulate feeding behavior and growth in fruit fly (Lee, Kwon, Lee, Kwon, Min, Jung, Kim, You, Tatar and 
Yu, 2008b; Lee, You, Choo, Han and Yu, 2004). However, those studies investigated the role of SNPF signaling by widespread overexpression. Therefore, sNPF action in specific circuits has not been addressed. To identify neurons that must express sNPF to mediate SMD, we first characterized the expression pattern of the SNPF-GAL4 driver using the reporter UAS-mCD8GFP (a cell membrane marker) together with UASRedStinger (a nuclear marker) (Figure $5 \mathrm{C}$ ), or UAS-Denmark (a dendritic marker) together with UAS-sytGFP (a presynaptic marker) (Figure S5D and S5E) (Nicolai, Ramaekers, Raemaekers, Drozdzecki, Mauss, Yan, Landgraf, Annaert and Hassan, 2010). This sNPF-GAL4 driver generates an expression pattern that recapitulates the pattern revealed by SNPF antibody immunocytochemistry (Nässel, Enell, Santos, Wegener and Johard, 2008). We identified more than 200 cells that were labeled by the sNPF-GAL4 driver, including MB neurons, s-LNv (white arrows in Figure S5E), a subset of PI (pars intercerebrails) neurons, neurons in the SOG (suboesophageal ganglion), and a subset of antenna lobe neurons specifically labeled in the male fly brain (white arrows in Figure 5C), indicating that some sNPF expressing cells are sexually dimorphic. We cannot exclude the possibility that some small sNPF-GAL4 labeled cells may have escaped detection.

Next we asked how sNPFR1, the only-known Drosophila sNPF receptor (NPFR76F; CG7395; sNPFR1) (Feng, Reale, Chatwin, Kennedy, Venard, Ericsson, Yu, Evans and Hall, 2003b; Mertens, Meeusen, Huybrechts, De Loof and Schoofs, 2002), mediates sNPF signaling to modulate SMD behavior. We used sNPFR1-RNAi combined with various GAL4 drivers to identify the neurons that express the SNPF receptor to mediate 
SMD behavior (Figure 5D). Expression of $s N P F R 1-R N A i$ in neuronal or glial populations eliminated SMD (elav and repo in Figure 5D), indicating that glial cells expressing sNPFR1 are also required for SMD behavior. SMD was intact when SNPFR1 expression was reduced in SNPF expressing cells via sNPF-GAL4 (SNPF in Figure 5D), indicating that SNPFR1 (receptor) expression among sNPF-positive cells, potentially for autocrine signaling, is not required for SMD behavior.

Next we tested GAL4 drivers that label different populations of clock neurons to identify the subset of clock neurons required for sNPFR1 signaling. Although SMD remained intact with expression of $s N P F R 1-R N A i$ in PDF-expressing neurons ( $p d f$ in Figure 5D), SMD was abolished by expression of sNPFR1-RNAi in CRY-positive cells (cry in Figure 5D). Thus, sNPFR1 expression in neurons that express CRY but not PDF is required for SMD. Expression of sNPFR1-RNAi in a subset of dorsal neurons (Clk4.1M in Figure 5D) did not eliminate SMD. This result implies that sNPFR1 expression is not required in the CLK-positive small subset of dorsal neurons to induce SMD behavior, even though sNPF expression in a subset of CLK-positive neurons is required for SMD (C/k4.1M in Figure 5B). Reducing sNPFR1 signaling in MB, EB, and FB neurons eliminated SMD (c547, MB247, ok107, and 14-94 in Figure 5D), indicating that sNPFR1 signaling in these memory circuits are required to elicit SMD.

To delineate the relationship between sNPF signaling and its known receptor sNPFR1, we performed rescue experiments using sNPFR1 100427 mutants which are homozygous-lethal. As expected, SMD was restored in sNPFR1 $1^{M 100427}$ mutants by 
expressing the UAS-sNPFR1 transgene via the sNPFR1-GAL4 driver (lane 3 in Figure 5E). Interestingly, expression of the UAS-sNPFR1 transgene via the sNPF-GAL4 driver in sNPFR $1^{M 100427}$ mutants rescued the lethality but not SMD behavior (lane 2 in Figure 5E). These studies reveal that sNPFR1 expression in sNPF-positive cells is crucial for survival, but not sufficient to fully elicit SMD behavior, indicating that sNPFR1 expression in regions other than sNPF-expressing cells are required to elicit intact SMD behavior.

To identify neurons that express sNPFR1 to mediate the SMD behavior, we characterized the expression pattern of the sNPFR1-GAL4 driver using the reporter UAS-mCD8GFP together with UAS-RedStinger (Figure 5F). We found more than 100 cells labeled by the sNPFR1-GAL4 driver including MB neurons and a subset of PI neurons. A subset of cells in the antenna lobe and cells located in the ventral optic lobe (white arrows in Figure 5F) were labeled only in male fly brains, indicating that a portion of sNPFR1 expressing cells are sexually dimorphic.

Since we already determined that Gr5a-positive sensory neurons are required for SMD (Figure 2 and 3), we next asked whether sNPF signaling in these sensory cells are also required for SMD. Previous studies have implicated sNPF signaling in gustatory sensory transduction (Ci, Wu and Su, 2014; Inagaki, Panse and Anderson, 2014). Expression of sNPF-RNAi in Gr5a-positive, but not Gr66a-positive cells, abolished SMD (Figure S5F), indicating that sNPF signaling in Gr5a-positive neurons is required to elicit SMD behavior. In addition, we found that the function of CLK/CYC in neurons mediating 
sNPF signaling is crucial for SMD (sNPF and sNPFRR1 in Figure 4C and D), indicating that SNPF signaling is connected to gustatory neural circuits and circadian clock neurons for modulating SMD behavior.

\section{Sexually dimorphic sNPF-expressing neurons are required for SMD}

Sexual dimorphism refers "the differences in appearance between males and females of the same species, such as color, shape, size, and structure, that are caused by the inheritance of one of the other sexual pattern in the genetic material". In Drosophila, the neural sex determination gene, fruitless (fru) has been identified and investigated. FRU is translated only in males to regulate the transcription of a set of downstream effectors. At least in neuronal populations, expression of FRU in female induces male-like behaviors (Yamamoto, 2007). We previously showed that FRU-positive, sexually dimorphic neuropeptide F (NPF) expressing neurons modulate LMD behavior (Kim, Jan and Jan, 2013).

As we have shown above, sNPF- and sNPFR1-GAL4 expression patterns exhibit sexual dimorphism (Figures 5C and 5F). We therefore wanted to identify the sexually dimorphic neurons among sNPF-positive cells. First, we used sNPF-GAL4 to identify sexually dimorphic cells using intersectional method with $f r u^{F L P}$ as described above (Figure 3B). Interestingly, we found $\sim 40$ FRU-positive and sNPF-positive neurons in the male brain (sNPF-GAL4 in Figure 6A). When sNPFR1-GAL4 was used to identify sexually dimorphic cells, strong GFP fluorescence near the antenna lobe was detected in the 
male brain (sNPFR1-GAL4 in Figure 6A). These data suggest the presence of FRUpositive cells among the sNPF- and sNPFR1-postive neuronal populations.

We then examined the function of these FRU-positive neurons on SMD behavior. To test whether the small subset of neurons positive for both fru and sNPF or positive for both fru and sNPFR1, are involved in SMD, we expressed tetanus toxin light chain (UAS[stop]TNT active) with sNPF- or sNPFR1-GAL4 drivers along with $f r u^{F L P}$ to inhibit synaptic transmission in sexually dimorphic subsets of fru-positive cells. Expression of UAS-TNT in fru- and sNPF-positive, or fru- and sNPFR1-positive, neurons, abolished SMD behavior (Figure 6B). As a control, we found that SMD was unaffected when we used each of these GAL4 drivers in combination with UAS[stop]TNT $T_{\text {inactive }}$ to express an inactive form of tetanus toxin light chain (Figure 6C). Similar results (Figure S6G) were obtained using the cell autonomous toxin Ricin A (UAS[stop]RicinA), which encodes only the catalytic subunit of the toxin that can enter the cell to cause cell death (Hidalgo, Urban and Brand, 1995). These data suggest that those FRU-positive neurons that are involved in SNPF signaling are crucial to elicit SMD behavior.

Next, we asked if only the masculinized form of those neurons can induce male-specific SMD behavior. Feminization of sNPF-GAL4 labeled neurons using UAS-tra ${ }^{F}$ expression eliminated SMD, suggesting that the male-specific function of sNPF-positive neurons is required to elicit SMD ( $N$ NPF in Figure 6D). Male flies did not show any significant courtship activity when sNPFR1-GAL4 labeled cells were feminized. These males failed to compete for a mate, and therefore we could not measure mating duration in these 
flies (sNPFR1 in Figure 6D). These results suggest that the male-specific function of a subset of sNPFR1-positive cells is critical to induce courtship behavior. In addition, we noticed that the expression of GFP fluorescence in SOG regions was lost when sNPFR1-positive cells were feminized via UAS-tra ${ }^{F}$ expression (compare Figure 5F with Figure S6F), raising the possibility that a subset of sNPFR1-positive cells that function in SOG regions is responsible for normal courtship behavior.

Next we used the dendritic marker UAS[stop]DscamGFP to label neurons positive for both fru and sNPF, or positive for both fru and sNPFR1. We found large dendritic arbors spanning from dorsal to SOG regions as revealed by using the SNPF-GAL4 driver (sNPF in Figure 6E). No GFP expressing processes positive for both fru and sNPFR1 could be found with the dendritic marker (Figure 6E), suggesting that the GFP fluorescence shown in Figure $6 \mathrm{~A}$ corresponds to presynaptic terminals rather than dendrites.

As described above, both sNPF-GAL4 and sNPFR1-GAL4 label thousands of cells in the fly brain. To further narrow down the population of SNPF- and sNPFR1-expressing neurons involved in SMD, we used different GAL80 lines to block the function of sNPFor sNPFR1-GAL4 drivers in brain regions with GAL80 expression. With these GAL4/GAL80 combinations, we knocked down sNPF or sNPFR1 expression using sNPF-RNAi or sNPFR1-RNAi respectively (Figure 6F and 6G). SMD was abolished when sNPF was knocked down using sNPF-GAL4 with cry-GAL80 that expresses GAL80 in CRY-positive neurons, with MB-GAL80 that expresses specifically in the 
mushroom body, or with both cry-GAL80 and MB-GAL80 (Figure 6F). This indicates that the function of sNPF signaling in CRY- and MB-positive neurons is not sufficient to fully induce SMD. Similar results were obtained when sNPFR1 was knocked down using sNPFR1-GAL4 with cry-GAL80 or MB-GAL80 (Figure 6G). We tested the expression of these GAL4/GAL80 combinations using UAS-mCD8GFP and UAS-RedStinger, confirming the suppression of GFP expression via GAL80 expression (Figures S6A-E). All these data suggest that sNPF/sNPFR1 signaling in multiple brain regions is required to generate SMD.

\section{Sexual experience affects the properties of synapses and activity of neurons involved in sNPF signaling}

The most probable place which reflects plastic changes in neurons are synapses, the junction between neurons. Various studies have revealed that the synaptic terminals of sensory and interneurons are pivotal sites of neuromodulation (Sweatt, 2016). In Drosophila, previous studies have shown that starvation increase the sugar sensitivity of gustatory neurons leading to altered behavioral responses to sugar (Gaudry and Kristan, 2009), which may involve the neuropeptide sNPF signaling that functions locally in olfactory tissues to modulate this neuronal plasticity (Root, Ko, Jafari and Wang, 2011). In this scenario, sNPF signaling can create internal states of starved or well-fed flies so that sensory inputs should be gated and modulated by those internal states (Kennedy, Asahina, Hoopfer, Inagaki, Jung, Lee, Remedios and Anderson, 2014). 
As shown above, we have demonstrated that SMD behavior displays behavioral plasticity, which indicates that males reversibly change the mating duration dependent on their environmental context (Figures 1 and S1). Thus we asked if we could find neuronal populations presenting synaptic plasticity dependent on male's sexual experiences. To selectively label nerve terminals, we used neuronal synaptobrevin-GFP chimera (nsybGFP), which allows visualizing the terminals of axonal projections. The expression of nsybGFP alters neither morphology nor circuitry of neurons thus it could be used as a tracer of axonal projections of neural circuits in the fly brain (Vosshall, Wong and Axel, 2000). Many studies have used nsybGFP to measure activitydependent chronic plasticity in olfactory circuits (Sachse, Rueckert, Keller, Okada, Tanaka, Ito and Vosshall, 2007) (Devaud, Acebes and Ferrus, 2001). Further, GFP fluorescence of nsybGFP allows visualization of synapse formation, retraction, and maturation during embryonic development or metamorphosis, and for determining projection patterns and target sites of CNS neurons in the adult brain (Estes, Ho, Narayanan and Ramaswami, 2000).

To look into the synaptic plasticity of the sNPF-expressing neuronal population, we used UAS[stop]nSybGFP with fru ${ }^{F L P}$ (Yu, Kanai, Demir, Jefferis and Dickson, 2010b) in combination with the sNPF-GAL4 driver to analyze the minimal neural populations functionally responsible for SMD behavior (Figure 6A), given that the sNPF-GAL4 driver labels numerous cells in the adult brain (compare Figure S7A with Figure 7A). Interestingly, the number of $n S y b G F P$ particles increased in experienced males (Figure 7B), while the average size of those particles was similar between naïve and 
experienced males (Figure 7C). The percent of area covered by nsybGFP particles increased in the brains from experienced males (Figure 7D). Interestingly, there was a large increase in the number and intensity of nsybGFP particles near SOG regions in experienced males (white circles in Figure 7A), indicating that gustatory inputs from female partners enhance sNPF signaling within specific CNS regions. We also used UAS[stop]DscamGFP with fru ${ }^{F L P}$ in combination with $S N P F-G A L 4$ to analyze how sexual experience modulates the dendritic morphology of responsible neurons (Figure S7B). The number of DscamGFP particles (Figure S7C), average size (Figure S7D), and percent of area (Figure S7E) were all increased in experienced males, indicating that chronic sexual experience modulates the dendritic morphology of sexually dimorphic sNPF-positive neurons responsible for inducing SMD behavior. We suggest that this neuronal reorganization in sNPF-expressing neurons alters the internal state of the male brain that enables the male's decision to terminate mating earlier than before.

Internal states of experienced male may be correlated with the number of synapses and morphology of dendrites. To find out whether neuronal activity is altered in neurons involved in SMD, we used the CaLexA (calcium-dependent nuclear import of LexA) system (Masuyama, Zhang, Rao and Wang, 2012), to measure neuronal activity based on the activity dependent nuclear import of a nuclear factor of activated T cells (NFAT). Since SMD requires chronic exposure to females for at least $12-24 \mathrm{~h}$, the repeated sensory inputs could conceivably result in the accumulation of the engineered transcription factor in the nuclei of active neurons in vivo. This system has been successfully applied to measure $\mathrm{Ca}^{2+}$ levels over the course of multiple hours inside the 
brain (Guo, Yu, Jung, Abruzzi, Luo, Griffith and Rosbash, 2016; Kayser, Yue and Sehgal, 2014b; Koh, He, Gorur-Shandilya, Menuz, Larter, Stewart and Carlson, 2014; Liu, Liu, Tabuchi and Wu, 2016; Park, Dus, Kim, Abu, Kanai, Rudy and Suh, 2016).

We first examined sNPF-expressing neurons, since they are a critical modulator of SMD behavior. As shown above, sexual experiences substantially alter the synaptic terminals and dendritic morphology of sNPF-positive neurons (Figure 7A and Figures S7A-B). Indeed, the neuronal activity of some sNPF-GAL4 labeled neurons was altered by sexual experience. Male flies harboring sNPF-GAL4 and LexAop-CD2-GFP; UASmLexA-VP16-NFAT, LexAop-CD8-GFP-2A-CD8-GFP (CaLexA) showed robust fluorescence in three dorsomedial neurons and one dorsolateral neuron after 1 day of sexual experience (labeled as a-d in Figure 7E). In contrast, one neuron in the lateral and one neuron in the ventromedial regions showed constant robust fluorescence in both naïve and experience males (labeled as e-f in Figure 7E). Thus, sexual experience increased the fluorescence signal of dorsal neurons compared to that of ventral neurons (abcd/ef fluorescence) in males (Figures 7F). GFP fluorescence in female brains showed different patterns, suggesting that it is the male-specific sNPF-positive neurons that are activated after sexual experience (bottom panels in Figure 7E). Male flies harboring sNPFR1-GAL4 and CaLexA showed diminished GFP fluorescence in ventromedial antennal lobe neurons, as compared to dorsomedial antennal lobe neurons, after 1 day of sexual experience (labeled as a-b in Figure S7F). Thus sexual experience decreased the fluorescence signal of ventromedial antennal lobe neurons compared to that of dorsomedial antennal lobe neurons (b/a fluorescence) in males 
(Figures S7G). As control, we examined PDF- and NPF-expressing neurons that are not involved in SMD behavior by using the pdf-GAL4 or npf-GAL4 driver to assess their neuronal activity. We did not find any significant changes in GFP fluorescence between PDF- and NPF-expressing neurons by sexual experience (Figures S7H-J), indicating that SNPF signaling but not PDF or NPF signaling is specifically involved in generating SMD behavior via sexual experience. 


\section{DISCUSSION}

Our study provides evidences that male flies invest less time for copulation when they are sexually satiated. Males retain a memory of sexual experience for several hours and economize mating duration accordingly. This behavior relies primarily on the gustatory input (Figure 2) that originates from female pheromones produced by oenocytes (Figure 1), indicating that contact chemoreception is required for SMD induction. Male-specific Gr5a-positive cells are sensory neurons required for recognizing the presence of females and these gustatory neurons are connected to the central brain region to process information for SMD behavior (Figure 3). We further show that the circadian clock genes Clk and cyc, but not per or tim, are important for SMD behavior. In addition, we found that the function of CLK/CYC in a subset of clock neurons within the circadian rhythm circuit is important for SMD behavior (Figure 4A-D). SMD requires the function of ellipsoid bodies and mushroom bodies within the CNS for memory processing, and this processing depends on orb2 function within the mushroom bodies (Figure 4E-F). We provide evidence for the crucial involvement of the neuropeptide sNPF, but not PDF or NPF, in the modulation of reproductive behavior by the male's prior sexual experience (Figure 5). Sexually dimorphic sNPF/sNPFR1-positive neurons are necessary to generate SMD (Figure 6). Remarkably, sexual experience alters the synaptic structure and the neuronal activity of a subset of neurons expressing sNPF likely reflecting neuronal modulation relevant for the SMD behavior (Figure 7).

\section{How does sNPF signaling orchestrate multiple functions of brain dynamics?}


Neuropeptides can function in a diverse way. In the central brain, they act as neuromodulators or cotransmitters. Neuropeptides also can play a role as circulating hormones via the hemolymph and as neuromediators when released by neurons to influence peripheral targets (Nassel and Winther, 2010). Neuropeptide sNPF is widely distributed in various types of neurons in the brain and VNC of the fly. It has been suggested that sNPF is a multifunctional neuropeptide (Nassel, Enell, Santos, Wegener and Johard, 2008). For example, the effect of sNPF on growth may be limited to a restricted subset of the sNPF-expressing neurons near insulin-producing cells in pars cerebrails $(\mathrm{PI})$. Thus the regulation of growth by sNPF signaling is only a part of a spectrum of sNPF functions in physiology and behavior in Drosophila (Lee, Kwon, Lee, Kwon, Min, Jung, Kim, You, Tatar and Yu, 2008a).

The multiple functions of sNPF in fruit fly have been suggested as follows: It plays a role in olfaction at the synapses between olfactory receptor neurons (ORN) and second order neurons in the antennal lobe. It functions in the mushroom bodies to process olfactory information and modulate olfactory learning to support the role of mushroom bodies. It also functions as a neuromodulator in the central complex of the fly brain to control locomotion. As described above, the upstream signal of the insulin-producing cells also functions as circulating neurohormones. Finally, it likely acts as modulator/cotransmitter functions in various brain circuits and the ventral nerve cord (Johard, Enell, Gustafsson, Trifilieff, Veenstra and Nassel, 2008). To understand the functional diversity of sNPF signaling in regulating various functions of brain dynamics, 
we need to interfere with the function of sNPF more precisely in discrete circuits. In this context, one might expect the various function of sNPF can be achieved by modulating its receptor expression in diverse neuronal types. There is only a single Drosophila sNPF receptor identified so far (NPFR76F; CG7395; sNPFR1) (Feng, Reale, Chatwin, Kennedy, Venard, Ericsson, Yu, Evans and Hall, 2003a; Mertens, Meeusen, Huybrechts, De Loof and Schoofs, 2002; Reale, Chatwin and Evans, 2004). It already has been postulated that this receptor can segregate the function of sNPF by coupling it to different signaling pathways (Feng, Reale, Chatwin, Kennedy, Venard, Ericsson, Yu, Evans and Hall, 2003a; Lee, Kwon, Lee, Kwon, Min, Jung, Kim, You, Tatar and Yu, 2008a; Mertens, Meeusen, Huybrechts, De Loof and Schoofs, 2002; Reale, Chatwin and Evans, 2004).

Consistent with previous reports, our study provides a new line of evidence that a small number of the neuronal population requires the function of SNPF to modulate SMD behavior (Figure 5B and Figure 6F). First, sNPF functions only in a neuronal population to elicit SMD behavior. Second, sNPF expression in neurons expressing its receptor is also necessary for SMD behavior. Third, the SNPF expression in PDF-positive neurons that function as the central pacemaker is dispensable for SMD behavior. Fourth, CRYpositive dorsal clock neurons and DN1 clock neurons are essential for sNPF signaling to induce intact SMD behavior. We suggest that the function of CLK/CYC in these neurons might connect the sNPF signaling to modulate timing-based decision making behavior. Fifth, sNPF signaling is necessary both in ellipsoid bodies and mushroom bodies, but not in the fan-shape bodies to elicit memory formation. These results 
suggest that there is a specialized subset of neurons that expresses sNPF, which specifically contributes to inducing SMD behavior.

We found interesting features by interfering with the function of the sNPF receptor sNPFR1 in precisely defined circuits (Figure 5C and Figure 6G). First, the expression of sNPFR1 both in neuronal and glial population is important to elicit SMD behavior. Second, the expression of sNPFR1 in sNPF-positive cells is dispensable for intact SMD behavior. Third, among clock neurons, PDF-positive central pacemaker cells and DN1 clock cells are dispensable. However, some of the CRY-positive neurons are important for SMD behavior properly. Fourth, SNPF signaling is necessary in all the tested memory circuits such as ellipsoid body, mushroom body and fan-shaped body.

Knocking down sNPFR1 in fan-shaped body neurons diminished SMD behavior. However, inactivation of fan-shaped body neurons using UAS-Kir2.1 had no effect. One scenario compatible with both findings is that SNPFR1 indirectly modulate the function of fan-shaped body neurons. The mechanism of modulating neuronal activity by neuropeptides can be classified in two different time scales. In short-term effects, neuropeptides can modify the activity of ion channels and alter the release of neurotransmitters or the synaptic response. In long-term effects, neuropeptides may signal by altering gene expression and strengthening synaptic transmission via regulating synaptic structures (Chen and Ganetzky, 2012).

It is also worth noting that the sNPFR1-expressing FB cells are not restricted to 
neuronal cell types. We speculate that glial cell population in this region could be substantial circuit participants to induce SMD behavior through sNPF signaling since sNPFR1 knock down in glial cell population also eliminates this behavior (repo in Figure 5D). However, we cannot exclude the possibility that other as yet unrecognized types of sNPFR1-positive FB cells are involved in SMD behavior as well.

The ability of sNFP knowdown but not Kir2.1 expression in FB cells to impact SMD behavior could also indicate that the sNFP mediated inhibition not activation of fanshape body neurons is crucial to SMD behavior. If the fan-shape body neurons responsible for SMD behavior are inhibitory neurons, forced depolarization of fan-shape body neurons will disrupt SMD behavior. To test this possibility, we expressed bacterial sodium channel UAS-NachBac to force fan-shaped body neurons into a depolarized state. Indeed, such ectopic activation of fan-shaped body neurons disrupted SMD behavior (Figure S4D). In summary, we suggest that sNPF signaling might reduce the neuronal activity of fan-shaped body neurons to induce intact SMD behavior.

As described above, we identified the neural circuits involving sNPF-positive and sNPFR1-positive neurons. In the case of sNPFR1-positive cells, we suggest that some of the glial cell population is required to elicit intact SMD behavior. We also identified the sexually dimorphic FRU-positive sNPF-expressing neurons that modulate this specific behavior (Figure 6). These sexually dimorphic sNPF-expressing neurons show synaptic plasticity depending on the male's sexual experience (Figure 7A-D and Figure S7B-E). Finally, these neurons alter the neuronal activities following the sexual experiences 
(Figure 7E-F and Figure S7F-G).

In this paper, we identified the new function of the SNPF in modulating timing-based decision-making process of a male fruit fly and we also found that circadian clock genes Clock/cycle are specifically involved with this process. These results raise the question as to how the SNPF signaling pathway is connected to clock pathways to modulate timing-based decision-making.

\section{How are Gr5a-positive gustatory inputs connected to SNPF signaling and the CLK/CYC clock networks to modulate mating duration?}

We have uncovered contact chemoreception by several male-specific gustatory neurons as a major source for gauging the male fruit fly sexual experience. We found that a male's sexual fulfillment is provided via gustatory but not olfactory inputs (Figure 2). The fly gustatory system appears to have a simple map compared to the olfactory system, which recognizes and distinguishes thousands of odors, suggesting that the fly may not need to finely distinguish many different tastes. There are two non-overlapping neural populations identified by either Gr5a or Gr66a expression. Gr5a cells recognize sugars and mediate acceptance/attractive behaviors, whereas Gr66a cells recognize bitter compounds and mediate avoidance behavior. In this respect, Gr5a cells can be designated as "acceptance" cells rather than "sweet" cells (Wang, Singhvi, Kong and Scott, 2004). Thus, the specific involvement of Gr5a but not Gr66a cells in SMD is consistent with the notion that both sugar and females will be categorized as 
acceptance signals. We also found that male-specific Gr5a cells detect the female presence and send signals to the central brain to induce SMD behavior (Figure 3). We then identified LUSH, the odorant binding protein, and SNMP1, the regulator of the pheromone receptor's signaling in Gr5a-positive cells, as critical components for eliciting the SMD behavior (Figure 2). Thus, we propose that the expression of snmp1 and lush in these Gr5a-positive cells can signal the existence of females via gustatory circuits.

In summary, we found that Gr5a-dependent contact chemoreception is involved in detecting female presence and connected to the sNPF signaling pathway (Figure S5). How are these two circuits connected? First, sNPF signaling has been implicated in regulating feeding behavior in Drosophila, similar to its mammalian counterpart NPY (Lee, Kwon, Lee, Kwon, Min, Jung, Kim, You, Tatar and Yu, 2008b). Moreover, indirect evidence suggests that SNPF signaling is required to modulate innate behavioral states such as satiety, which can be shaped by gustatory stimuli to alter neural circuits (Ci, Wu and Su, 2014). Future studies will reveal how Gr5a-positive sensory neurons are connected to sNPF-positive neural circuits. Uncovering the feedback interactions between Gr5a-positive cells and sNPF signaling circuits will shed light on how the information from the sensory modalities can be transmitted to the neuropeptidergic system and integrated into changing internal states of brain dynamics.

There are several lines of evidence indicating that circadian clock genes, especially Clk and cyc, regulate feeding (Chatterjee, Tanoue, Houl and Hardin, 2010; Krupp and 
Levine, 2010; Sarov-Blat, So, Liu and Rosbash, 2000; Xu, Zheng and Sehgal, 2008).

For example, the input of neuronal clocks and clocks in metabolic tissues are coordinated to mediate effective energy homeostasis with gustatory inputs via Clk and cyc (Xu, Zheng and Sehgal, 2008). The clock tunes the gustatory system to a higher gain level in the morning that allows the fly to temporally couple the morning bout of activity with food-detection machinery (Chatterjee, Tanoue, Houl and Hardin, 2010). Interestingly, cyc function supplied via the Gr5a-GAL4 driver can rescue the fooddetection deficit, which suggests that the perceived meaning of a sensory input is determined not just by the modality or intensity of the stimulus, but also by the circadian time when the stimulus is registered (Chatterjee, Tanoue, Houl and Hardin, 2010). In addition, it was reported that $C l k$ and $c y c$ specifically regulate starvation-induced sleeploss, indicating a close relationship between CLK/CYC function and gustatorydependent metabolic homeostasis (Keene, Duboue, McDonald, Dus, Suh, Waddell and Blau, 2010).

The relationship between SNPF signaling and CLK/CYC function could derive from their common link to the insulin-signaling pathway. Insulin signaling regulates growth, metabolism, and aging of animals, including Drosophila melanogaster. Drosophila sNPF signaling regulates growth by ERK-mediated insulin signaling (Lee, Kwon, Lee, Kwon, Min, Jung, Kim, You, Tatar and Yu, 2008b). Insulin also interacts with the sNPF signaling pathway by acting as a satiety signal to decrease sNPFR expression, and in turn decrease motivated feeding (Root, Ko, Jafari and Wang, 2011). Drosophila FOXO is known to regulate metabolism and oxidative stress. FOXO regulates insulin activity 
through feedback mechanisms, and also affects circadian rhythms (Zheng, Yang, Yue, Alvarez and Sehgal, 2007). FOXO is expressed predominantly in the fat body, suggesting that foxo has non-cell-autonomous effects on central circadian clock function. We propose a possible role of sNPF connecting FOXO-mediated insulin signaling to central and peripheral clock networks. In support of this notion, a previous study demonstrates that the gustatory-dependent metabolic state of a fly influences the clock to modulate behavioral rhythms via insulin and sNPF signaling pathways (Erion and Sehgal, 2013). Finding the possible links among these circuits would be an interesting subject for future investigation.

\section{Neural reuse model: how the same clock neurons modulate both circadian and interval timing-based behaviors.}

It is common for neural circuits established for one purpose to be exapted during evolution (exaptation) (Gould and Vrba, 1982; Skoyles, 1999) for different uses without losing their original function. 'Neural reuse' theories can explain the evolutionarydevelopmental properties of neural plasticity involved in human cognition such as reuse of motor control circuits for language, reuse of motor control circuits for memory, and reuse of circuits for numerical cognition (Anderson, 2010). Neural reuse may be more prevalent in insect brains since many insects possess small brains that have been miniaturized during evolution. Their small size suggests that insects are under selective pressure to reduce energetic costs and brain size. Thus, in smaller brains, there may be an increased prevalence of neural reuse (Chittka and Niven, 2009; Niven and Chittka, 
2010).

In support of this hypothesis, it has been proposed that dCLK/CYC-controlled transcriptional/translational feedback loops operate differently in subsets of pacemaker neurons (Lee, Cho, Kang, Jeong, Chen, Yoo and Kim, 2016). Thus the same CLK/CYCcontrolled molecular pathways may contribute to the specific functions dependent on the different neural circuits. Consistent with this hypothesis, we previously showed that male flies use a subset of PDF/NPF-expressing clock neurons, which were originally established to regulate circadian rhythm, for processing the information of previous exposure to rivals (Kim, Jan and Jan, 2013). Here we propose another evidence of neural reuse model found in the fly brain to modulate both circadian and interval timingbased behaviors. Our studies suggest that neural reuse occurs in the brain of a fruit fly similar to that of humans (Anderson, 2010; Dumont and Robertson, 1986; Skoyles, 1999).

\section{Possible relationship between SMD and LMD}

Our earlier study found that previous exposure to rivals lengthens mating duration, a behavior named Longer-Mating-Duration (LMD) (Kim, Jan and Jan, 2012). Even though LMD and SMD share a common behavioral phenotype in regulating mating duration, there are clear differences in the underlying mechanisms (Table 1-Move this table from supplemental material to the main text as it nicely compare LMD with SMD). First, LMD requires only visual input while SMD requires multiple inputs, including gustatory input. 
Second, LMD requires the function of the clock genes PER/TIM, whereas SMD requires the function of CLK/CYC. Third, LMD requires the EB for its memory storage, but SMD requires both the $\mathrm{EB}$ and $\mathrm{MB}$. Fourth, rut and amn are important to process the memory for LMD, while orb2 is critical for SMD. Fifth, LMD requires the function of sexually dimorphic LNd clock neurons, however SMD requires that of Gr5a-positive sensory neurons. Thus both behaviors are involved in modulating the length of copulation but use different genetic components and different but partially overlapping neuronal circuitry.

These two behavioral circuits might have evolved independently since they use different sensory cues for detecting 'rivals vs. females' for 'sexual competition vs. sexual satiety'. Male flies depend on visual input to recognize rivals (Kim, Jan and Jan, 2012), however they depend on female pheromones via contact chemoreception to recognize female presence and register sexual experience (Figures 1 and 2). Recent studies demonstrating the need of males to touch the female body in order to persistently induce female-oriented behaviors also support this premise (Agrawal, Safarik and Dickinson, 2014; Kohatsu and Yamamoto, 2015). We propose that contact chemoreception induced by female body pheromones contributes to the determination of a male's mating investment in Drosophila melanogaster. Like LMD (Kim, Jan and Jan, 2012), SMD requires clock genes involved in non-circadian functions, such as learning and memory, habituation, sleep, drug sensitization, and mating duration (Hall, 2005), without the feedback between PER/TIM and CLK/CYC that is crucial for circadian rhythm. 
Even though LMD and SMD use different sensory modalities and neuropeptide expressing neurons, that information needs to be collected together and sent to output neurons for the termination of mating. It has been reported that mating termination involves neuropeptide corazonin (Crz) expressing neurons, subset of dopaminergic and GABAergic neurons (Crickmore and Vosshall, 2013; Tayler, Pacheco, Hergarden, Murthy and Anderson, 2012). Further investigation will be required to find possible connections between LMD and SMD circuits with the output neural circuitry.

\section{Both SMD and LMD provide a social behavior model using fruit fly}

As noted by Darwin, the brain of social insects is the most marvelous atoms of matter in the world more so than the brain of man (Darwin, 1871), considering how the remarkably complex neural circuits which process social information are integrated into such a tiny space (Chittka and Niven, 2009). Unlike the classic eusocial insects such as ants and bees, flies are not typically regarded for their group dynamics, however, this view is changing with growing evidence of social interactions in Drosophila (Battesti, Moreno, Joly and Mery, 2012; Benzer, 1967; Billeter, Jagadeesh, Stepek, Azanchi and Levine, 2012; Billeter, Atallah, Krupp, Millar and Levine, 2009; Billeter and Levine, 2013; Bretman, Fricke and Chapman, 2009; Ganguly-Fitzgerald, Donlea and Shaw, 2006; Garbaczewska, Billeter and Levine, 2013; Kacsoh, Bozler, Hodge, Ramaswami and Bosco, 2015; Kacsoh, Bozler, Ramaswami and Bosco, 2015; Kent, Azanchi, Smith, Formosa and Levine, 2008; Kim, Jan and Jan, 2012, 2013; Krupp, Kent, Billeter, 
Azanchi, So, Schonfeld, Smith, Lucas and Levine, 2008; Krupp and Levine, 2010; Lefranc, Jeune, Thomas-Orillard and Danchin, 2001; Levine, Funes, Dowse and Hall, 2002; Lewis, Heys, Prescott and Lizé, 2014; Loyau, Blanchet, Van Laere, Clobert and Danchin, 2012; Mery, Varela, Danchin, Blanchet, Parejo, Coolen and Wagner, 2009; Saltz and Foley, 2011; Sarin and Dukas, 2009; Schneider, Dickinson and Levine, 2012; Tinette, Zhang and Robichon, 2004; Vosshall, 2007; Wang and Anderson, 2010; Wang, Han, Mehren, Hiroi, Billeter, Miyamoto, Amrein, Levine and Anderson, 2011a). Moreover, a recent study suggests that Drosophila melanogaster exhibits collective behavior that has been investigated in schooling fish, flocking birds and human crowds (Ramdya, Lichocki, Cruchet, Frisch, Tse, Floreano and Benton, 2015). It is clear that social communication is actually much more fundamental to life than previously considered (Kacsoh, Bozler, Ramaswami and Bosco, 2015). Thus, the fly is a useful model system as a compromise between tractability and richness, to study the conserved neural circuits that elicit social behavior (Burne, Scott, van Swinderen, Hilliard, Reinhard, Claudianos, Eyles and McGrath, 2011; Olsen and Wilson, 2008; Vosshall, 2007). Together with recent studies of social experience-mediated and context-dependent behavior of fruit flies, LMD and SMD will help us understand how animals receive social information and then process it at the molecular level.

\section{SMD behavior provides a novel and genetically traceable satiety paradigm}

Satiety is usually defined as the state of being fed beyond capacity. Along this line, satiety studies were focusing on feeding behaviors in Drosophila (Krashes, DasGupta, 
Vreede, White, Armstrong and Waddell, 2009; Nassel and Williams, 2014; Schleyer, Saumweber, Nahrendorf, Fischer, von Alpen, Pauls, Thum and Gerber, 2011; Söderberg, Carlsson and Nässel, 2012). In this context, the distinctive feature of satiation defined as the blunted sensitivity to food tastes, especially to sugars. In flies, myoinhibitory peptide (MIP) has been identified as an important factor to coordinate feeding motivation and maintain a constant body weight (Min, Chae, Jang, Choi, Lee, Jeong, Jones, Moon, Kim and Chung, 2016; Min and Chung, 2016). The drosulfakinin (DSK) peptide has been identified as satiety signals together with insulin-like peptides (DILPs) (Soderberg, Carlsson and Nassel, 2012). DSK is a cholecystokinin-like peptide found in the fruit fly, and cholecystokinin signaling appears well conserved during evolution. Like sNPF, DSK is a multifunctional neuropeptide that regulates gut function, satiety and food ingestion, hyperactivity and aggression, and synaptic plasticity during neuromuscular junction and development (Nassel and Williams, 2014).

Sexual satiety defines the long-term inhibition of masculine sexual behavior after repeated ejaculations in rats and mice. By measuring the c-Fos expression level in the brain regions of the sexually satiated male, researchers identified several areas in the male rat forebrain of relevance to sexual satiety (Phillips-Farfan and Fernandez-Guasti, 2007). Recently Zhang and colleagues reported that Drosophila males show reproductive satiety (Zhang, Rogulja and Crickmore, 2016). In their behavioral paradigm, males' mating drive gets reduced after 4 hours of exposure to excessive females. The major difference between this report and ours is that Zhang and colleagues did not specifically measure the mating duration. Instead, they measured all 
the mating behaviors during the 4 hours of time frame and interpreted the reduced mating performance as satiety index. DopR1-mediated superior medial protocerebrum (SMPA) dopaminergic circuits has been identified as an important element for the mating drive.

Here we demonstrate novel forms of satiety paradigm related to sexual experience, not to food ingestion. We found that sexually satiated male Drosophila reduces the investment on mating (Figure 1). Surprisingly, gustatory neurons crucial for sugar detection also detect female pheromone to register the satiety states in the central brain (Figure 2). Since ILP, DSK, and MIP have been reported as modulators of food-related satiety signaling, future study will investigate the relationship between the brain circuitry we found and the ILP/DSK circuits (Min, Chae, Jang, Choi, Lee, Jeong, Jones, Moon, Kim and Chung, 2016; Min and Chung, 2016; Nassel and Williams, 2014; Soderberg, Carlsson and Nassel, 2012). This investigation will help us identify the fundamental principles regarding how food ingestion-related satiety and sexual satiety signaling share the neural circuits and still maintain their respective independent functionality. It will also be interesting to investigate the role of dopaminergic circuits in SMD behavior since dopamine plays a crucial role to modulate reproductive satiety of male fruit fly (Zhang, Rogulja and Crickmore, 2016).

In summary, we report a novel anorexigenic pathway that controls satiety related to sexual experiences in Drosophila. This novel paradigm will provide a new avenue to study how the brain actively maintains a constant mating drive to invest on sexual 
behavior properly.

\section{ACKNOWLEDGEMENTS}

We thank Dr. Kweon Yu (Korea Research Institute of Bioscience and Biotechnology:

KRIBB, Korea) for kindly providing valuable sNPF-related fly strains. We thank Dr. Joshua Bagley (UCSF) and Dr. Kyeongjin Kang (Sungkyungkwan University, Korea) for helpful comments on this paper. The work was supported by NIH grant 2R37NS040929 to YNJ. LYJ and YNJ are investigators of Howard Hughes Medical Institute. 


\section{FIGURE LEGENDS}

Figure 1. General characteristics of 'Shorter-Mating-Duration (SMD)' behaviour. (A) Naïve males were kept for 5 days after eclosion in groups of 4 males. Experienced males were kept for 4 days after eclosion in groups then experienced with 5 virgin females 1 day before assay; for detailed methods, see EXPERIMENTAL PROCEDURES. (B) Mating duration (MD) assays of Canton-S (CS), Oregon-R, WTBerlin and w1118 males. White boxes represent naïve males and grey boxes represent experienced ones. (C) MD assays of CS males experienced with females in different time duration. Different female-experienced times are described below the boxes. (D) MD assays of CS males after isolated from female experience. Males were reared with sufficient numbers of virgin females for $2 \mathrm{~h}$ to be assured having sexual experience then isolated. Assay times after isolation are described below the boxes. Boxes labeled naïve and exp. represent standard rearing conditions described in Figure 1A. (E) MD assay of CS males experienced with different female strains 1 day before assay. Experienced females' genotypes are described below the boxes. To make mated females $\left(f e^{\text {mated }}\right)$, 4day-old 10 CS virgin females were placed with 5-day-old 20 CS males for 6 hours then separated to an empty vials. These females were used for experienced females 1 day

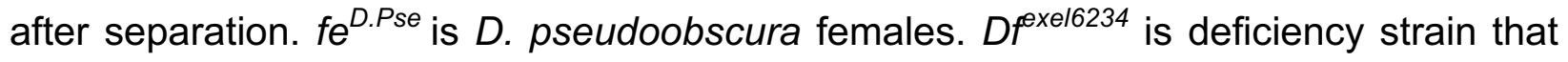
lacks sex-peptide receptor (SPR) expression, the critical component for post-mating switch in female Drosophila (Yapici, Kim, Ribeiro and Dickson, 2008). To make virgin females behave as mated females ( $f r u^{U A S-m S P}$ ), flies expressing UAS-mSP (membrane bound form of male sex-peptide) were crossed with flies expressing fru-GAL4 driver as 
described previously (Yang, Rumpf, Xiang, Gordon, Song, Jan and Jan, 2009). To make oenocyte-deleted females (oenocyte (-)), flies expressing UAS-Hid/rpr virgins were crossed with flies expressing tub-GAL80ts; oeno-GAL4 males then the female progenies were kept in $22^{\circ} \mathrm{C}$ for 3 days. Flies were moved to $29^{\circ} \mathrm{C}$ for 2 days before assay to express UAS-Hid/rpr to kill the oenocytes in these females. The oeno-GAL4 (PromE(800)-GAL4) was described previously (Wang, Han, Mehren, Hiroi, Billeter, Miyamoto, Amrein, Levine and Anderson, 2011b). To make oenocytes-masculinized females (oenotra-RNAi), flies expressing UAS-tra-RNAi were crossed with oeno-GAL4 driver. To test whether genotypes of female partners during MD assay affect MD, oenocyte masculinized females (oeno ${ }^{\text {tra-RNAi}}$ ) were used. Box plots represent the median value (horizontal line inside box), the mean value ('+' symbol inside box), interquartile range (height of the box, $50 \%$ of the data within this range), and minimum and maximum value (whiskers). Asterisks represent significant differences revealed by Student's $t$ test $\left({ }^{*} p<0.05,{ }^{* *} p<0.01,{ }^{* * *} p<0.001\right)$. The same notations for statistical significance are used in other figures. Number signs represent significant differences revealed by Dunn's Multiple Comparison Test $\left({ }^{\#} p<0.05\right)$. The same symbols for statistical significance are used in all other figures. See EXPERIMENTAL PROCEDURES for detailed statistical analysis used in this study.

Figure 2. Pheromone sensing proteins expressed in Gr5a-positive neurons are responsible to induce SMD. (A) MD assay of various vision, olfactory, and auditory mutants. Genotypes are indicated below the graphs. To test vision is required for SMD, 
CS males were reared and sexually experienced in constant dark for entire 5 days (dark). (B) MD assays for GAL4 driven cell death of which labels sweet cells (Gr5a) or bitter cells (Gr66a) using UAS-Hid/rpr. (C) Brains of flies expressing Gr5a-GAL4 or Gr66a-GAL4 together with UAS-mCD8GFP, UAS-RedStinger were immunostained with anti-GFP (green), anti-DsRed (red) and nc82 (blue) antibodies. Scale bars represent $100 \mu \mathrm{m}$ in the $1^{\text {st }}, 2^{\text {nd }}$, and $4^{\text {th }}$ panels, $10 \mu \mathrm{m}$ in the $3^{\text {rd }}$ panels from the left. White and yellow boxes indicate the magnified regions of interest presented next right panels. The $3^{\text {rd }}$ panels from the left are presented as grey scale for clearly showing the axon projection patterns of gustatory neurons in the adult subesophageal ganglion (SOG) labeled by GAL4 drivers. (D) MD assays for GAL4 mediated knockdown of LUSH via UAS-Iush-IR; UAS-dicer (lush-RNAi). Names of the GAL4 drivers are indicated below the graphs. (E) Mating duration assays of snmp $1^{1}$ rescue experiments. Genotypes are indicated above the graphs.

Figure 3. Male specific FRU-positive subsets of Gr5a-positive neurons are required to generate SMD. (A) Front legs and proboscis of flies expressing Gr5a- and Gr66a- GAL4 drivers together with UAS-RedStinger; UAS[stop]mCD8GFP; fru ${ }^{F L P}$ were imaged live under fluorescent microscope. Yellow arrows indicate fruitless-positive neurons. Scale bars represent $10 \mu \mathrm{m}$. (B) Brains of flies expressing Gr5a-GAL4 or Gr66a-GAL4 together with UAS[stop]mCD8GFP; $f u^{F L P}$ were immunostained with anti-GFP (green) and nc82 (magenta) antibodies. White and yellow boxes indicate the magnified regions of interest presented next right panels. The right panels are shown grey scale for clearly 
presenting the axon projection patterns of gustatory neurons in the adult SOG labeled by GAL4 drivers. Scale bars represent $100 \mu \mathrm{m}$. (C) Same experiment of Figure 3B using presynaptic marker UAS[stop]n-sybGFP; fru ${ }^{F L P}$. (D) MD assays of Gr5a- and Gr66a-GAL4 drivers for inactivation of synaptic transmission of male-specific neurons among each GAL4-labeld neurons via UAS[stop]TNTactive; fru ${ }^{F L P}$. (E) Control experiments of Figure 3D with inactive form of UAS-TNT using UAS[stop]TNTinactive; $\mathrm{fru}^{F L P}$. (F) Mating duration assays for Gr5a- and Gr66a-GAL4 drivers for feminization of neurons via UAS-tra ${ }^{F}$.

Figure 4. Circadian clock genes CLK and CYC are specifically involved with SMD. (A) MD assays of circadian clock mutants. The genotypes specified below the graphs. (B) $\mathrm{MD}$ assays of $c y c^{01}$ rescue experiments. Genotypes are indicated above the graphs. (C), (D) MD assay for GAL4 mediated knockdown of CLK or CYC via UAS-CIk-IR; UASdicer (CIk-RNAi) or UAS-cyc-IR; UAS-dicer (cyc-RNAi). Names of the GAL4 drivers are indicated below the graphs. (E) MD assays of memory mutants. The genotypes specified below the graphs. (F) MD assay for GAL4 mediated knockdown of ORB2 via UAS-orb2-IR; UAS-dicer (orb2-RNAi). Names of the GAL4 drivers are indicated below the graphs.

Figure 5. sNPF signaling is required to produce SMD. (A) MD assays of neuropeptides and neuropeptides receptor mutants. The genotypes specified below the graphs. (B) MD assays for GAL4 mediated knockdown of SNPF via UAS-sNPF-IR; UAS-dicer (sNPF-RNAi). Names of the GAL4 drivers are indicated below the graphs. (C) Brains of 
flies expressing sNPF-GAL4 together with UAS-mCD8GFP; UAS-RedStinger were immunostained with anti-GFP (green), anti-DsRed (red) and nc82 (blue) antibodies. White boxes indicate the magnified regions of interest presented next bottom panels. Scale bars represent $100 \mu \mathrm{m}$. (D) MD assays for GAL4 mediated knockdown of sNPFR1 via UAS-sNPF-R-IR; UAS-dicer (sNPF-R-RNAi). Names of the GAL4 drivers are indicated below the graphs. (E) MD assays of $s N P F-R^{M I 004271}$ rescue experiments. Genotypes are indicated below the graphs. (F) Brains of flies expressing sNPF-R-GAL4 together with UAS-mCD8GFP; UAS-RedStinger were immunostained with anti-GFP (green), anti-DsRed (red) and nc82 (blue) antibodies. White boxes indicate the magnified regions of interest presented next bottom panels. Scale bars represent 100 $\mu \mathrm{m}$.

Figure 6. Both male-specific and redundant sNPF signaling modulates SMD. (A) Brains of flies expressing sNPF-GAL4 or sNPF-R-GAL4 together with UAS[stop]mCD8GFP; $\mathrm{fru}^{F L P}$ were immunostained with anti-GFP (green) and nc82 (magenta) antibodies. White boxes indicate the magnified regions of interest presented the bottom panels. Scale bars represent $100 \mu \mathrm{m}$. (B) MD assays of sNPF- and sNPF-R-GAL4 drivers for inactivation of synaptic transmission of male-specific neurons among each GAL4labeled neurons via UAS[stop]TNTactive; fru ${ }^{F L P}$. (C) Control experiments of Figure 6B with inactive form of UAS-TNT using UAS[stop]TNTinactive; fru ${ }^{F L P}$. (D) MD assays for sNPF- and sNPF-R-GAL4 drivers for feminization of neurons via UAS-tra ${ }^{F}$. Since feminizing sNPF-R-GAL4 labeld neurons seriously affect courtship activity, MD assays 
could not be done. (E) Brains of flies expressing sNPF-GAL4 or sNPF-R-GAL4 together with UAS[stop]DscamGFP; fru ${ }^{F L P}$ were immunostained with anti-GFP (green) and nc82 (magenta) antibodies. White boxes indicate the magnified regions of interest presented the bottom panels. Dashed circles in represent the clearly disappeared GFP signals compared to Figure 6A (white arrows). Scale bars represent $100 \mu \mathrm{m}$. (F) and (G) MD assays for GAL4/GAL80 mediated knockdown of SNPF or sNPF-R1 via UAS-sNPF-IR; UAS-dicer (sNPF-RNAi) or UAS-sNPF-R-IR; UAS-dicer (sNPF-R-RNAi). Names of the GAL4/GAL80 drivers' combination are indicated below the graphs.

Figure 7. Sexual experience alters the number of synapses and the neural activity of neuropeptide expressing neurons. (A) Flies expressing sNPF-GAL4 with UAS[stop]nsybGFP; fru ${ }^{F L P}$ were naively reared (upper panels) or experienced with virgin females 1 day before dissection (bottom panels). Brains of flies were immunostained with antiGFP (red hot) and nc82 (grey) antibodies. GFP is pseudocolored "red hot". Dashed circles represent the clearly increased GFP signals compared to upper panel. Scale bars represent $100 \mu \mathrm{m}$. (B) Number of particles shown in (A) was quantified using ImageJ software. (C) Average size of particles shown in (A) was quantified. (D) Percent of brain area covered by particles shown in $(A)$ are quantified. See EXPERIMENTAL PROCEDURES for details. (E) Brains of naïve male (top), experienced male (middle), and female (bottom) flies expressing sNPF-GAL4 along with LexAop-CD2-GFP; UASmLexA-VP16-NFAT, LexAop-CD8-GFP-2A-CD8-GFP were dissected then 
immunostained with anti-GFP (green) and anti-nc82 (magenta) antibodies. GFP is pseudocolored "fire" in the middle and the right panels. Warmer colors reflect increased signal intensity. Cells showing strong GFP signals were labeled "a-f" from the top to bottom. White arrows indicate the subset of neurons showing strong GFP signals; dashed circles indicate the subset of neurons showing reduced GFP signals compared to the different rearing condition. (F) GFP fluorescence of "a-d" regions is normalized by "e-f" region. See EXPERIMENTAL PROCEDURES for detailed quantification methods.

\section{EXPERIMENTAL PROCEDURES}

\section{Fly Rearing and Strains}

Drosophila melanogaster were raised on cornmeal-yeast medium at similar densities to yield adults with similar body sizes. Flies were kept in $12 \mathrm{~h}$ light: $12 \mathrm{~h}$ dark cycles (LD) at $25^{\circ} \mathrm{C}$ ( $\mathrm{ZT} 0$ is the beginning of the light phase, $\mathrm{ZT} 12$ beginning of the dark phase) except for some experimental manipulation (experiments with the flies carrying tub$\left.G A L 80^{t s}\right)$. Wild-type flies were Canton-S. To reduce the variation from genetic background, all flies were backcrossed for at least 3 generations to CS strain. All mutants and transgenic lines used here have been described previously.

We are very grateful to the colleagues who provided us with many of the lines used in this study. We obtained the following lines from Dr. Joel D. Levine and Joshua J. Krupp (University of Toronto, Canada): PromE(800)-GAL4 (oeno-GAL4 in this study); from Dr. Kweon Yu (Korea Research Institute of Bioscience and Biotechnology: KRIBB, Korea), 
sNPF-GAL4, UAS-sNPF, sNPF-RNAi, sNPF-R-RNAi; from Dr. Barry Dickson (HHMI Janelia Research Campus, USA): UAS[stop]mCD8GFP; fru ${ }^{F L P}$, UAS[stop]DscamGFP; $\mathrm{fru}^{F L P}, U A S\left[\right.$ stop]nsybGFP; fru ${ }^{F L P}$, UAS[stop]TNT $T_{\text {active; }}$ fru ${ }^{F L P}$, UAS[stop]TNT inactive; fru $^{F L P}$, UAS[stop]RicinA; fru ${ }^{F L P}$, fru-GAL4, orb2 ${ }^{\perp}$, orb2 ${ }^{\triangle Q}$; from Dr. Chung-Hui Yang (Duke University Medical Center, USA): Gr5a-GAL4, elav-GAL80; from Dr. Toshiro Aigaki (Tokyo Metropoitan University, Japan): UAS-mSP; from Dr. Herman Wijnen (University of Virginia, USA) (Goda, Mirowska, Currie, Kim, Rao, Bonilla and Wijnen, 2011): UAScyc; cyc ${ }^{01}$, elav ${ }^{c 155} ;$ UAS-cyc; cyc ${ }^{01}$, elav ${ }^{c 155} ;$ pdf-GAL80; cyc ${ }^{01}$, elav ${ }^{c 155} ;$ tub-GAL80 ${ }^{\text {ts }}$; $\operatorname{cyc}^{01}$; from Dr. Martin Heisenberg (Universität Würzburg, Germany): WT Berlin, ninaE ${ }^{17}$; from Dr. Alex C. Keene (University of Nevada, USA) and Dr. Justin Blau (New York University, USA): $c y c^{01}, C^{J}{ }^{J r k}, c r y-G A L 80 ; c r y-G A L 80$, tim-GAL4, pdf-GAL4, cry-GAL4; from Dr. Ravi Allada (Northwestern University, USA): Clk4.1M-GAL4; from Dr. Amita Sehgal (University of Pennsylvania Medical School, USA: pdf ${ }^{01}$, per $^{01}$ tim $^{01}$, per ${ }^{01}$,tim ${ }^{01}$; from Dr. J. Douglas Armstrong (University of Edinburgh): c547-GAL4; from Dr. Jing Wang (University of California San Diego, USA): UAS-mLexA-VP16-NFAT, LexAopCD2-GFP; LexAop-CD8-GFP-2A-CD8-GFP; from Dr. Gero Miesenböck (University of Oxford, UK): MB-GAL80; from Dr. Ulrike Heberlein (HHMI Janelia Research Campus): 14-94-GAL4; Dr. Chun Han and Dr. Peter Soba generated the following lines from our laboratory: UAS-CD4-tdGFP, UAS-CD4-tdTomato.

The following lines were obtained from Bloomington Stock Center (\#stock number): $\operatorname{Orco}^{1}(\# 23129), \operatorname{Orco}^{2}(\# 23130)$, dnc $^{1}(\# 6020)$, rut $^{2080}$ (\#9405), amn ${ }^{1}(\# 5954)$, UAS- 
tubGAL80 ${ }^{t s}$ (\#7018), UAS-Kir2.1 (\#6596), UAS-NachBac (\#9469), Df(1)Exel6234 (\#7708), UAS-tra ${ }^{F}$ (\#4590), GustD ${ }^{\times 6}$ (\#8607), Gr66a-GAL4 (\#28801), UAS-mCD8GFP (\#5130), UAS-RedStinger (\#8547), snmp 1 ${ }^{1}$ (\#25043), snmp12 (\#25042), UAS-snmp1

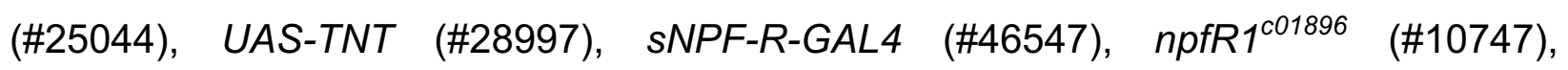
Df(3R)BSC464 (npfR1 deficiency line, \#24968), sNPF-R ${ }^{M 100427}$ (\#30996), elav ${ }^{c 155}$ (\#458), repo-GAL4 (\#7415), UAS-dicer (\#24650, \#24651), UAS-Denmark; UAS-syteGFP (\#33064), UAS-n-sybGFP (\#6921), npf-GAL4 (\#25682), tra-RNAi (\#28512), lush-RNAi (\#31657), orb2-RNAi (\#27050); from Kyoto Stock Center (DGRC): iav ${ }^{1}$ (\#101174), ok107-GAL4 (\#106098), GMR-Hid (\#108419); from Vienna Stock Center (VDRC): ClkRNAi (\#v42833, \#v42834), cyc-RNAi (\#v11765); from Harvard Exelixis Collection: sNPF ${ }^{c 00448}(\# \mathrm{c} 00448)$.

\section{Mating Duration Assays}

Mating duration assay was performed as previously described (Kim, Jan and Jan, 2012). For naïve males, 4 males from the same strain were placed into a vial with food for 5 days. For experienced males, 4 males from the same strain were placed into a vial with food for 4 days then eight CS virgin females were introduced into vials for last 1 day before assay. Five CS females were collected from bottles and placed into a vial for 5 days. These females provide both sexually experienced partners and mating partners for mating duration assays. At the fifth day after eclosion, males of the appropriate strain and CS virgin females were mildly anaesthetized by $\mathrm{CO}_{2}$. After placing a single female in to the mating chamber, we inserted a transparent film then placed a single male to the other side of the film in each chamber. After allowing for $1 \mathrm{~h}$ of recovery in the 
mating chamber in a $25^{\circ} \mathrm{C}$ incubator, we removed the transparent film and recorded the mating activities. Only those males that succeeded to mate within $1 \mathrm{~h}$ were included for analyses. Initiation and completion of copulation were recorded with an accuracy of 10 sec, and total mating duration was calculated for each couple. All assays were performed from noon to $4 \mathrm{pm}$.

\section{Courtship assays}

Courtship assay was performed as previously described (Ejima and Griffith, 2007), under normal light conditions in circular courtship arenas $11 \mathrm{~mm}$ in diameter, from noon to $4 \mathrm{pm}$. Courtship latency is the time between female introduction and the first obvious male courtship behavior such as orientation coupled with wing extensions. Once courtship began, courtship index was calculated as the fraction of time a male spent in any courtship-related activity during a 10 min period or until mating occurred. Mating initiation is the time after male flies successfully mounted on female.

\section{Locomotion assays}

For climbing assay, individual flies were placed in a $15 \mathrm{ml}$ falcon tube (Fisher Scientific) and were gently tapped to the bottom of the tube. The time taken for the flies to climb 8 $\mathrm{cm}$ of the tube wall was recorded. Each fly was tested 5 times. Other than a single instance, all flies were seen to reach the target height within 2 min, which was the experimental cut-off time. Velocity was obtained by dividing the lines $(\mathrm{mm})$ a fly crossed (distance walked) by time (sec) a fly reached the line of the tube. For horizontal (spontaneous) locomotor activities, a single fly was first brought to the middle of the 
column by gentle shaking and then the fly movement was constantly monitored for 5 min and recorded. Total fraction of time flies walked during 5 min was calculated and number of stops during 5 min was also counted then calculated (Mohammad, Singh and Sharma, 2009).

\section{Immunostaining and antibodies}

As described before (Lee and Luo, 1999), brains dissected from adults 5 days after eclosion were fixed in $4 \%$ formaldehyde for $30 \mathrm{~min}$ at room temperature, washed with 1\% PBT three times (30 min each) and blocked in 5\% normal donkey serum for $30 \mathrm{~min}$. The brains were then incubated with primary antibodies in $1 \%$ PBT at $4{ }^{\circ} \mathrm{C}$ overnight followed with fluorophore-conjugated secondary antibodies for 1 hour at room temperature. Brains were mounted with anti-fade mounting solution (Invitrogen, catalog \#S2828) on slides for imaging. Primary antibodies: chicken anti-GFP (Aves Labs, 1:1000), rabbit anti-DsRed express (Clontech, 1:250), mouse anti-Bruchpilot (nc82) (DSHB, 1:50), mouse anti-PDF (DSHB, 1:100). Fluorophore-conjugated secondary antibodies: Alexa Fluor 488-conjugated goat anti-chicken (Invitrogen, 1:100), Alexa Fluor 488-conjugated donkey anti-rabbit (Invitrogen, 1:100), RRX-conjugated donkey anti-rabbit (Jackson Lab, 1:100), RRX-conjugated donkey anti-mouse (Jackson Lab, 1:100), Dylight 649-conjugated donkey anti-mouse (Jackson Lab, 1:100).

\section{Imaging and quantitative analysis of GFP Fluorescence}

As described before (Kayser, Yue and Sehgal, 2014a), brains were visualized with a TCS SP2 confocal microscope and images processed with ImageJ (National Institutes 
of Health, Bethesda, MD; http://rsb.info.nih.gov/ij). All settings were kept constant between experimental conditions. Images were taken in $0.5 \mu \mathrm{M}$ steps unless otherwise specified. For CaLexA experiments, GFP fluorescence for CaLexA was normalized to nc82 staining, and then the fluorescence of ROI was quantified using the histogram tool of ImageJ. Both hemispheres of six fly brains were analyzed (total 12) for statistical analysis. For quantifying $n$-sybGFP particles, we used the "analyze particles" function of ImageJ. Average size represents the average size of analyzed particles. Percent of area represents percent of area that is covered with particles normalized by total area. All imaging and analysis were done blind to experimental condition.

\section{Statistical Analysis}

Statistical analysis of mating duration assay was described previously (Kim, Jan and Jan, 2012). More than 36 males (naïve or experienced) were used for mating duration assay. Our experience suggests that the relative mating duration differences between naïve and experienced condition are always consistent; however, both absolute values and the magnitude of the difference in each strain can vary. So we always include internal controls for each treatment as suggested by previous studies (Bretman, Westmancoat, Gage and Chapman, 2011). Therefore, statistical comparisons were made between groups that were naively reared or sexually experienced within each experiment. As mating duration of males showed normal distribution (KolmogorovSmirnov tests, $p>0.05)$, we used two-sided Student's $t$ tests. Each figure shows the mean \pm standard error (s.e.m) $\left(^{* * *}=p<0.001,{ }^{* *}=p<0.01,{ }^{*}=p<0.05\right)$. All analysis was done in GraphPad (Prism). Individual tests and significance are detailed in figure 
legends.

When we compare the difference of mating duration in experiments without internal control built in (Figure 1C and 1D), we always performed control experiments of wild type for each independent experiment for internal comparison. And in this case, we analyzed data using ANOVA for statistically significant differences (at a 95.0\% confidence interval) between the means of mating duration for all conditions. If a significant difference between the means was found by Kruskal-Wallis test, then the Dunn's Multiple Comparison Test was used to compare the mean mating duration of each condition to determine which conditions were significantly different from condition of interest. $\left({ }^{\#}=p<0.05\right)$ 


\section{REFERENCES}

Agrawal, S., Safarik, S., and Dickinson, M. (2014). The relative roles of vision and chemosensation in mate recognition of Drosophila melanogaster. J Exp Biol 217, 27962805.

Allada, R., and Chung, B.Y. (2010). Circadian organization of behavior and physiology in Drosophila. Annual review of physiology 72, 605-624.

Anderson, M.L. (2010). Neural reuse: a fundamental organizational principle of the brain. The Behavioral and brain sciences 33, 245-266; discussion 266-313.

Battesti, M., Moreno, C., Joly, D., and Mery, F. (2012). Spread of social information and dynamics of social transmission within Drosophila groups. Current Biology 22, 309-313.

Belote, J.M., and Baker, B.S. (1987). Sexual behavior: its genetic control during development and adulthood in Drosophila melanogaster. Proc Natl Acad Sci U S A 84, 8026-8030.

Benton, R., Vannice, K.S., and Vosshall, L.B. (2007). An essential role for a CD36related receptor in pheromone detection in Drosophila. Nature 450, 289-293.

Benzer, S. (1967). Behavioral mutants of Drosophila isolated by countercurrent distribution. Proc Natl Acad Sci U S A 58, 1112.

Billeter, J.-C., Jagadeesh, S., Stepek, N., Azanchi, R., and Levine, J.D. (2012). Drosophila melanogaster females change mating behaviour and offspring production based on social context. Proceedings of the Royal Society B: Biological Sciences 279, 2417-2425.

Billeter, J.C., Atallah, J., Krupp, J.J., Millar, J.G., and Levine, J.D. (2009). Specialized cells tag sexual and species identity in Drosophila melanogaster. Nature 461, 987-991.

Billeter, J.C., and Levine, J.D. (2013). Who is he and what is he to you? Recognition in Drosophila melanogaster. Curr Opin Neurobiol 23, 17-23.

Block, R.A., and Grondin, S. (2014). Timing and time perception: A selective review and commentary on recent reviews. Front Psychol 5, 648.

Bretman, A., Fricke, C., and Chapman, T. (2009). Plastic responses of male Drosophila melanogaster to the level of sperm competition increase male reproductive fitness. Proc Biol Sci 276, 1705-1711. Epub 2009 Feb 1725.

Bretman, A., Gage, M.J., and Chapman, T. (2011). Quick-change artists: male plastic behavioural responses to rivals. Trends in ecology \& evolution 26, 467-473.

Bretman, A., Westmancoat, J.D., and Chapman, T. (2013). Male control of mating duration following exposure to rivals in fruitflies. J Insect Physiol 59, 824-827.

Bretman, A., Westmancoat, J.D., Gage, M.J., and Chapman, T. (2011). Males use multiple, redundant cues to detect mating rivals. Curr Biol 21, 617-622.

Buhusi, C.V., and Meck, W.H. (2005). What makes us tick? Functional and neural mechanisms of interval timing. Nature reviews Neuroscience 6, 755-765.

Burke, C.J., Huetteroth, W., Owald, D., Perisse, E., Krashes, M.J., Das, G., Gohl, D., Silies, M., Certel, S., and Waddell, S. (2012). Layered reward signalling through octopamine and dopamine in Drosophila. Nature 492, 433-437.

Burne, T., Scott, E., van Swinderen, B., Hilliard, M., Reinhard, J., Claudianos, C., Eyles, D., and McGrath, J. (2011). Big ideas for small brains: what can psychiatry learn from worms, flies, bees and fish? Molecular psychiatry 16, 7-16. 
Byrne, P.G., and Rice, W.R. (2006a). Evidence for adaptive male mate choice in the fruit fly Drosophila melanogaster. Proceedings Biological sciences / The Royal Society 273 , 917-922.

Byrne, P.G., and Rice, W.R. (2006b). Evidence for adaptive male mate choice in the fruit fly Drosophila melanogaster. P R Soc B 273, 917-922.

Chatterjee, A., Tanoue, S., Houl, J.H., and Hardin, P.E. (2010). Regulation of gustatory physiology and appetitive behavior by the Drosophila circadian clock. Curr Biol 20, 300309.

Chen, X., and Ganetzky, B. (2012). A neuropeptide signaling pathway regulates synaptic growth in Drosophila. J Cell Biol 196, 529-543.

Chittka, L., and Niven, J. (2009). Are bigger brains better? Curr Biol 19, R995-R1008.

$\mathrm{Ci}, \mathrm{H}$., Wu, N., and Su, Y. (2014). Clock gene modulates roles of OXTR and AVPR1b genes in prosociality. PloS one 9, e109086.

Cook, T., Pichaud, F., Sonneville, R., Papatsenko, D., and Desplan, C. (2003). Distinction between color photoreceptor cell fates is controlled by Prospero in Drosophila. Dev Cell 4, 853-864.

Cordts, R., and Partridge, L. (1996). Courtship reduces longevity of maleDrosophila melanogaster. Animal Behaviour 52, 269-278.

Crickmore, M.A., and Vosshall, L.B. (2013). Opposing dopaminergic and GABAergic neurons control the duration and persistence of copulation in Drosophila. Cell 155, 881893.

Darwin, C. (1871). The descent of man, and selection in relation to sex. In Nineteenth Century Collections Online (NCCO): Science, Technology, and Medicine: 1780-1925 (London: J. Murray,), pp. 1 online resource (2 v.).

Demerec, M., and Kaufman, B.P. (1941). Time required for Drosophila males to exhaust the supply of mature sperm. American Naturalist, 366-379.

Demerec, M., and Kaufmann, B.P. (1941). Time required for Drosophila males to exhaust the supply of mature sperm. American Naturalist 75, 366-379.

Devaud, J.M., Acebes, A., and Ferrus, A. (2001). Odor exposure causes central adaptation and morphological changes in selected olfactory glomeruli in Drosophila. $J$ Neurosci 21, 6274-6282.

Dewsbury, D.A. (1982). Ejaculate cost and male choice. American Naturalist, 601-610.

Dissel, S., Angadi, V., Kirszenblat, L., Suzuki, Y., Donlea, J., Klose, M., Koch, Z., English, D., Winsky-Sommerer, R., van Swinderen, B., et al. (2015). Sleep restores behavioral plasticity to Drosophila mutants. Curr Biol 25, 1270-1281.

Dowling, D.K., and Simmons, L.W. (2012). Ejaculate economics: testing the effects of male sexual history on the trade-off between sperm and immune function in Australian crickets. PLoS One 7, e30172.

Dumont, J.P., and Robertson, R.M. (1986). Neuronal circuits: an evolutionary perspective. Science 233, 849-853.

Ejima, A., and Griffith, L.C. (2007). Measurement of Courtship Behavior in Drosophila melanogaster. CSH Protoc 2007, pdb prot4847.

Erion, R., and Sehgal, A. (2013). Regulation of insect behavior via the insulin-signaling pathway. Frontiers in physiology 4, 353.

Estes, P.S., Ho, G.L., Narayanan, R., and Ramaswami, M. (2000). Synaptic localization 
and restricted diffusion of a Drosophila neuronal synaptobrevin--green fluorescent protein chimera in vivo. J Neurogenet 13, 233-255.

Feng, G., Reale, V., Chatwin, H., Kennedy, K., Venard, R., Ericsson, C., Yu, K., Evans, P.D., and Hall, L.M. (2003a). Functional characterization of a neuropeptide F-like receptor from Drosophila melanogaster. Eur J Neurosci 18, 227-238.

Feng, G., Reale, V., Chatwin, H., Kennedy, K., Venard, R., Ericsson, C., Yu, K., Evans, P.D., and Hall, L.M. (2003b). Functional characterization of a neuropeptide F-like receptor from Drosophila melanogaster. Eur J Neurosci 18, 227-238.

Fernandez, M.P., and Kravitz, E.A. (2013). Aggression and courtship in Drosophila: pheromonal communication and sex recognition. J Comp Physiol A Neuroethol Sens Neural Behav Physiol 199, 1065-1076.

Ganguly-Fitzgerald, I., Donlea, J., and Shaw, P.J. (2006). Waking experience affects sleep need in Drosophila. Science 313, 1775-1781.

Garbaczewska, M., Billeter, J.C., and Levine, J.D. (2013). Drosophila melanogaster males increase the number of sperm in their ejaculate when perceiving rival males. Journal of insect physiology 59, 306-310.

Gaudry, Q., and Kristan, W.B., Jr. (2009). Behavioral choice by presynaptic inhibition of tactile sensory terminals. Nat Neurosci 12, 1450-1457.

Goda, T., Mirowska, K., Currie, J., Kim, M.H., Rao, N.V., Bonilla, G., and Wijnen, H. (2011). Adult circadian behavior in Drosophila requires developmental expression of cycle, but not period. PLoS genetics 7, e1002167.

Golombek, D.A., Bussi, I.L., and Agostino, P.V. (2014). Minutes, days and years: molecular interactions among different scales of biological timing. Philos Trans R Soc Lond B Biol Sci 369, 20120465.

Gould, S.J., and Vrba, E.S. (1982). Exaptation-a missing term in the science of form. Paleobiology, 4-15.

Gowaty, P.A., Steinichen, R., and Anderson, W.W. (2003). Indiscriminate females and choosy males: within- and between-species variation in Drosophila. Evolution 57, 20372045.

Griffith, L.C., and Ejima, A. (2009). Courtship learning in Drosophila melanogaster: Diverse plasticity of a reproductive behavior. Learn Memory 16, 743-750.

Guo, F., Yu, J., Jung, H.J., Abruzzi, K.C., Luo, W., Griffith, L.C., and Rosbash, M. (2016). Circadian neuron feedback controls the Drosophila sleep--activity profile. Nature 536, 292-297.

Hall, J.C. (2005). Systems approaches to biological rhythms in Drosophila. Methods Enzymol 393, 61-185.

Hasemeyer, M., Yapici, N., Heberlein, U., and Dickson, B.J. (2009). Sensory neurons in the Drosophila genital tract regulate female reproductive behavior. Neuron 61, 511-518.

Heisenberg, M. (2003). Mushroom body memoir: from maps to models. Nat Rev Neurosci 4, 266-275.

Hidalgo, A., Urban, J., and Brand, A.H. (1995). Targeted ablation of glia disrupts axon tract formation in the Drosophila CNS. Development 121, 3703-3712.

Inagaki, H.K., Panse, K.M., and Anderson, D.J. (2014). Independent, reciprocal neuromodulatory control of sweet and bitter taste sensitivity during starvation in Drosophila. Neuron 84, 806-820. 
Jenett, A., Schindelin, J.E., and Heisenberg, M. (2006). The Virtual Insect Brain protocol: creating and comparing standardized neuroanatomy. BMC bioinformatics 7 , 544.

Johard, H.A., Enell, L.E., Gustafsson, E., Trifilieff, P., Veenstra, J.A., and Nassel, D.R. (2008). Intrinsic neurons of Drosophila mushroom bodies express short neuropeptide F: relations to extrinsic neurons expressing different neurotransmitters. J Comp Neurol 507, 1479-1496.

Kacsoh, B.Z., Bozler, J., Hodge, S., Ramaswami, M., and Bosco, G. (2015). A Novel Paradigm for Non-Associative Long-Term Memory in Drosophila: Predator-Induced Changes in Oviposition Behavior. Genetics.

Kacsoh, B.Z., Bozler, J., Ramaswami, M., and Bosco, G. (2015). Social communication of predator-induced changes in Drosophila behavior and germline physiology.

Kayser, M.S., Yue, Z., and Sehgal, A. (2014a). A critical period of sleep for development of courtship circuitry and behavior in Drosophila. Science 344, 269-274.

Kayser, M.S., Yue, Z., and Sehgal, A. (2014b). A critical period of sleep for development of courtship circuitry and behavior in Drosophila. Science (New York, NY) 344, 269-274.

Keene, A.C., Duboue, E.R., McDonald, D.M., Dus, M., Suh, G.S., Waddell, S., and Blau, J. (2010). Clock and cycle limit starvation-induced sleep loss in Drosophila. Curr Biol 20, 1209-1215.

Keleman, K., Kruttner, S., Alenius, M., and Dickson, B.J. (2007). Function of the Drosophila CPEB protein Orb2 in long-term courtship memory. Nat Neurosci 10, 15871593.

Kennedy, A., Asahina, K., Hoopfer, E., Inagaki, H., Jung, Y., Lee, H., Remedios, R., and Anderson, D.J. (2014). Internal States and Behavioral Decision-Making: Toward an Integration of Emotion and Cognition. Cold Spring Harb Symp Quant Biol 79, 199-210.

Kent, C., Azanchi, R., Smith, B., Formosa, A., and Levine, J.D. (2008). Social context influences chemical communication in D. melanogaster males. Curr Biol 18, 1384-1389. Kim, M.S., Repp, A., and Smith, D.P. (1998). LUSH odorant-binding protein mediates chemosensory responses to alcohols in Drosophila melanogaster. Genetics 150, 711721.

Kim, W.J., Jan, L.Y., and Jan, Y.N. (2012). Contribution of visual and circadian neural circuits to memory for prolonged mating induced by rivals. Nat Neurosci 15, 876-883.

Kim, W.J., Jan, L.Y., and Jan, Y.N. (2013). A PDF/NPF Neuropeptide Signaling Circuitry of Male Drosophila melanogaster Controls Rival-Induced Prolonged Mating. Neuron 80, 1190-1205.

Koh, T.W., He, Z., Gorur-Shandilya, S., Menuz, K., Larter, N.K., Stewart, S., and Carlson, J.R. (2014). The Drosophila IR20a Clade of lonotropic Receptors Are Candidate Taste and Pheromone Receptors. Neuron 83, 850-865.

Kohatsu, S., and Yamamoto, D. (2015). Visually induced initiation of Drosophila innate courtship-like following pursuit is mediated by central excitatory state. Nature communications 6, 6457 .

Krashes, M.J., DasGupta, S., Vreede, A., White, B., Armstrong, J.D., and Waddell, S. (2009). A neural circuit mechanism integrating motivational state with memory expression in Drosophila. Cell 139, 416-427.

Krupp, J.J., Kent, C., Billeter, J.C., Azanchi, R., So, A.K., Schonfeld, J.A., Smith, B.P., 
Lucas, C., and Levine, J.D. (2008). Social experience modifies pheromone expression and mating behavior in male Drosophila melanogaster. Curr Biol 18, 1373-1383.

Krupp, J.J., and Levine, J.D. (2010). Biological rhythms: the taste-time continuum. Curr Biol 20, R147-149.

Lee, E., Cho, E., Kang, D.H., Jeong, E.H., Chen, Z., Yoo, S.H., and Kim, E.Y. (2016). Pacemaker-neuron-dependent disturbance of the molecular clockwork by a Drosophila CLOCK mutant homologous to the mouse Clock mutation. Proc Natl Acad Sci U S A.

Lee, K.-S., Kwon, O.-Y., Lee, J.H., Kwon, K., Min, K.-J., Jung, S.-A., Kim, A.-K., You, K.H., Tatar, M., and Yu, K. (2008a). Drosophila short neuropeptide F signalling regulates growth by ERK-mediated insulin signalling. Nat Cell Biol 10, 468-475.

Lee, K.S., Kwon, O.Y., Lee, J.H., Kwon, K., Min, K.J., Jung, S.A., Kim, A.K., You, K.H., Tatar, M., and Yu, K. (2008b). Drosophila short neuropeptide $F$ signalling regulates growth by ERK-mediated insulin signalling. Nat Cell Biol 10, 468-475.

Lee, K.S., You, K.H., Choo, J.K., Han, Y.M., and Yu, K. (2004). Drosophila short neuropeptide $F$ regulates food intake and body size. J Biol Chem 279, 50781-50789.

Lee, T., and Luo, L. (1999). Mosaic analysis with a repressible cell marker for studies of gene function in neuronal morphogenesis. Neuron 22, 451-461.

Lefevre, G., Jr., and Jonsson, U.B. (1962). Sperm transfer, storage, displacement, and utilization in Drosophila melanogaster. Genetics 47, 1719-1736.

Lefranc, A., and Bundgaard, J. (2000). The influence of male and female body size on copulation duration and fecundity in Drosophila melanogaster. Hereditas 132, 243-247.

Lefranc, A., Jeune, B., Thomas-Orillard, M., and Danchin, É. (2001). Non-independence of individuals in a population of Drosophila melanogaster: effects on spatial distribution and dispersal. Comptes Rendus de l'Académie des Sciences-Series III-Sciences de la Vie 324, 219-227.

Levine, J.D., Funes, P., Dowse, H.B., and Hall, J.C. (2002). Resetting the circadian clock by social experience in Drosophila melanogaster. Science 298, 2010-2012.

Lewis, Z., Heys, C., Prescott, M., and Lizé, A. (2014). You are what you eat: Gut microbiota determines kin recognition in Drosophila. Gut microbes 5, 541-543.

Li, Z., Ni, J.D., Huang, J., and Montell, C. (2014). Requirement for Drosophila SNMP1 for rapid activation and termination of pheromone-induced activity. PLoS genetics 10, e1004600.

Liu, G., Seiler, H., Wen, A., Zars, T., Ito, K., Wolf, R., Heisenberg, M., and Liu, L. (2006). Distinct memory traces for two visual features in the Drosophila brain. Nature 439, 551556.

Liu, S., Liu, Q.L., Tabuchi, M., and Wu, M.N. (2016). Sleep Drive Is Encoded by Neural Plastic Changes in a Dedicated Circuit. Cell 165, 1347-1360.

Louâpre, P., van Alphen, J.J.M., and Pierre, J.-S. (2010). Humans and insects decide in similar ways. PLoS ONE 5, e14251.

Louis, M., Huber, T., Benton, R., Sakmar, T.P., and Vosshall, L.B. (2008). Bilateral olfactory sensory input enhances chemotaxis behavior. Nat Neurosci 11, 187-199.

Loyau, A., Blanchet, S., Van Laere, P., Clobert, J., and Danchin, E. (2012). When not to copy: female fruit flies use sophisticated public information to avoid mated males. Scientific reports 2 .

Lupold, S., Manier, M.K., Ala-Honkola, O., Belote, J.M., and Pitnick, S. (2011). Male 
Drosophila melanogaster adjust ejaculate size based on female mating status, fecundity, and age. Behavioral Ecology 22, 184-191.

Makki, R., Cinnamon, E., and Gould, A.P. (2014). The development and functions of oenocytes. Annu Rev Entomol 59, 405-425.

Masuyama, K., Zhang, Y., Rao, Y., and Wang, J.W. (2012). Mapping neural circuits with activity-dependent nuclear import of a transcription factor. J Neurogenet 26, 89-102.

McKean, K.A., and Nunney, L. (2001). Increased sexual activity reduces male immune function in Drosophila melanogaster. Proc Natl Acad Sci U S A 98, 7904-7909.

Mertens, I., Meeusen, T., Huybrechts, R., De Loof, A., and Schoofs, L. (2002). Characterization of the short neuropeptide $\mathrm{F}$ receptor from Drosophila melanogaster. Biochemical and biophysical research communications 297, 1140-1148.

Mery, F., Varela, S.A.M., Danchin, É., Blanchet, S., Parejo, D., Coolen, I., and Wagner, R.H. (2009). Public versus personal information for mate copying in an invertebrate. Current Biology 19, 730-734.

Min, S., Chae, H.S., Jang, Y.H., Choi, S., Lee, S., Jeong, Y.T., Jones, W.D., Moon, S.J., Kim, Y.J., and Chung, J. (2016). Identification of a Peptidergic Pathway Critical to Satiety Responses in Drosophila. Curr Biol 26, 814-820.

Min, S., and Chung, J. (2016). Identification of a neural pathway governing satiety in Drosophila. BMB Rep 49, 137-138.

Mohammad, F., Singh, P., and Sharma, A. (2009). A Drosophila systems model of pentylenetetrazole induced locomotor plasticity responsive to antiepileptic drugs. BMC systems biology $3,11$.

Mucignat-Caretta, C. (2014). Neurobiology of chemical communication (CRC Press).

Nassel, D.R., Enell, L.E., Santos, J.G., Wegener, C., and Johard, H.A. (2008). A large population of diverse neurons in the Drosophila central nervous system expresses short neuropeptide F, suggesting multiple distributed peptide functions. BMC neuroscience 9 , 90.

Nässel, D.R., Enell, L.E., Santos, J.G., Wegener, C., and Johard, H.A.D. (2008). A large population of diverse neurons in the Drosophila central nervous system expresses short neuropeptide $F$, suggesting multiple distributed peptide functions. BMC neuroscience 9 , 90.

Nassel, D.R., and Williams, M.J. (2014). Cholecystokinin-Like Peptide (DSK) in Drosophila, Not Only for Satiety Signaling. Front Endocrinol (Lausanne) 5, 219.

Nassel, D.R., and Winther, A.M. (2010). Drosophila neuropeptides in regulation of physiology and behavior. Prog Neurobiol 92, 42-104.

Nicolai, L.J., Ramaekers, A., Raemaekers, T., Drozdzecki, A., Mauss, A.S., Yan, J., Landgraf, M., Annaert, W., and Hassan, B.A. (2010). Genetically encoded dendritic marker sheds light on neuronal connectivity in Drosophila. Proc Natl Acad Sci U S A 107, 20553-20558.

Nitabach, M.N., and Taghert, P.H. (2008). Organization of the Drosophila circadian control circuit. Current biology : CB 18, R84-93.

Niven, J.E., and Chittka, L. (2010). Reuse of identified neurons in multiple neural circuits. Behavioral and Brain Sciences 33, 285-285.

Olsen, S.R., and Wilson, R.I. (2008). Cracking neural circuits in a tiny brain: new approaches for understanding the neural circuitry of Drosophila. Trends in 
neurosciences $31,512-520$.

online, B. (2016). sexual dimorphism. In Encyclopædia Britannica Online (Encyclopædia Britannica Inc.,).

Pan, Y., Zhou, Y., Guo, C., Gong, H., Gong, Z., and Liu, L. (2009). Differential roles of the fan-shaped body and the ellipsoid body in Drosophila visual pattern memory. Learn Mem 16, 289-295.

Park, J.Y., Dus, M., Kim, S., Abu, F., Kanai, M.I., Rudy, B., and Suh, G.S. (2016). Drosophila SLC5A11 Mediates Hunger by Regulating $\mathrm{K}(+)$ Channel Activity. Curr Biol 26, 1965-1974.

Parker, G.A. (1970). Sperm competition and its evolutionary consequences in the insects. Biological Reviews 45, 525-567.

Parker, G.A. (1974). Courtship persistence and female-guarding as male time investment strategies. Behaviour 48, 157-183.

Parker, G.A. (1984). Sperm Competition and the Evolution of Animal Mating Strategies. In Sperm Competition and the Evolution of Animal Mating Systems, pp. 1-60.

Parker, G.A., and Pizzari, T. (2010). Sperm competition and ejaculate economics. Biol Rev Camb Philos Soc 85, 897-934.

Partridge, L., and Farquhar, M. (1981). Sexual activity reduces lifespan of male fruitflies. Nature 294, F582.

Phillips-Farfan, B.V., and Fernandez-Guasti, A. (2007). c-Fos expression related to sexual satiety in the male rat forebrain. Physiol Behav 91, 609-619.

Ramdya, P., Lichocki, P., Cruchet, S., Frisch, L., Tse, W., Floreano, D., and Benton, R. (2015). Mechanosensory interactions drive collective behaviour in Drosophila. Nature 519, 233-236.

Reale, V., Chatwin, H.M., and Evans, P.D. (2004). The activation of G-protein gated inwardly rectifying $\mathrm{K}+$ channels by a cloned Drosophila melanogaster neuropeptide Flike receptor. Eur J Neurosci 19, 570-576.

Rezaval, C., Pavlou, H.J., Dornan, A.J., Chan, Y.B., Kravitz, E.A., and Goodwin, S.F. (2012). Neural circuitry underlying Drosophila female postmating behavioral responses. Curr Biol 22, 1155-1165.

Rodrigues, V., Sathe, S., Pinto, L., Balakrishnan, R., and Siddiqi, O. (1991). Closely linked lesions in a region of the $X$ chromosome affect central and peripheral steps in gustatory processing in Drosophila. Molecular \& general genetics : MGG 226, 265-276.

Root, C.M., Ko, K.I., Jafari, A., and Wang, J.W. (2011). Presynaptic facilitation by neuropeptide signaling mediates odor-driven food search. Cell 145, 133-144.

Ryner, L.C., Goodwin, S.F., Castrillon, D.H., Anand, A., Villella, A., Baker, B.S., Hall, J.C., Taylor, B.J., and Wasserman, S.A. (1996). Control of male sexual behavior and sexual orientation in Drosophila by the fruitless gene. Cell 87, 1079-1089.

Sachse, S., Rueckert, E., Keller, A., Okada, R., Tanaka, N.K., Ito, K., and Vosshall, Leslie B. (2007). Activity-Dependent Plasticity in an Olfactory Circuit. Neuron 56, 838850.

Saleem, S., Ruggles, P.H., Abbott, W.K., and Carney, G.E. (2014). Sexual Experience Enhances Drosophila melanogaster Male Mating Behavior and Success. PloS one 9, e96639.

Saltz, J.B., and Foley, B.R. (2011). Natural genetic variation in social niche construction: 
social effects of aggression drive disruptive sexual selection in Drosophila melanogaster. The American Naturalist 177, 645-654.

Sarin, S., and Dukas, R. (2009). Social learning about egg-laying substrates in fruitflies. Proceedings of the Royal Society of London B: Biological Sciences 276, 4323-4328.

Sarov-Blat, L., So, W.V., Liu, L., and Rosbash, M. (2000). The Drosophila takeout gene is a novel molecular link between circadian rhythms and feeding behavior. Cell 101, 647-656.

Schleyer, M., Saumweber, T., Nahrendorf, W., Fischer, B., von Alpen, D., Pauls, D., Thum, A., and Gerber, B. (2011). A behavior-based circuit model of how outcome expectations organize learned behavior in larval Drosophila. Learn Memory 18, 639653.

Schneider, J., Dickinson, M.H., and Levine, J.D. (2012). Social structures depend on innate determinants and chemosensory processing in Drosophila. Proceedings of the National Academy of Sciences 109, 17174-17179.

Siegel, R.W., and Hall, J.C. (1979). Conditioned responses in courtship behavior of normal and mutant Drosophila. Proc Natl Acad Sci U S A 76, 3430-3434.

Skoyles, J.R. (1999). Neural plasticity and exaptation.

Soderberg, J.A., Carlsson, M.A., and Nassel, D.R. (2012). Insulin-Producing Cells in the Drosophila Brain also Express Satiety-Inducing Cholecystokinin-Like Peptide, Drosulfakinin. Front Endocrinol (Lausanne) 3, 109.

Söderberg, J.A.E., Carlsson, M.A., and Nässel, D.R. (2012). Insulin-producing cells in the Drosophila brain also express satiety-inducing cholecystokinin-like peptide, Drosulfakinin. Frontiers in Endocrinology 3.

Sweatt, J.D. (2016). Neural Plasticity \& Behavior - Sixty Years of Conceptual Advances. J Neurochem.

Tayler, T.D., Pacheco, D.A., Hergarden, A.C., Murthy, M., and Anderson, D.J. (2012). A neuropeptide circuit that coordinates sperm transfer and copulation duration in Drosophila. Proc Natl Acad Sci U S A 109, 20697-20702.

Tinette, S., Zhang, L., and Robichon, A. (2004). Cooperation between Drosophila flies in searching behavior. Genes, Brain and Behavior 3, 39-50.

Tucci, V. (2011). Sleep, circadian rhythms, and interval timing: evolutionary strategies to time information. Front Integr Neurosci 5, 92.

Verzijden, M.N., ten Cate, C., Servedio, M.R., Kozak, G.M., Boughman, J.W., and Svensson, E.I. (2012). The impact of learning on sexual selection and speciation. Trends in ecology \& evolution $27,511-519$.

Vosshall, L.B. (2007). Into the mind of a fly. Nature 450, 193-197.

Vosshall, L.B., Wong, A.M., and Axel, R. (2000). An Olfactory Sensory Map in the Fly Brain. Cell 102, 147-159.

Wang, L., and Anderson, D.J. (2010). Identification of an aggression-promoting pheromone and its receptor neurons in Drosophila. Nature 463, 227-231.

Wang, L., Han, X., Mehren, J., Hiroi, M., Billeter, J.-C., Miyamoto, T., Amrein, H., Levine, J.D., and Anderson, D.J. (2011a). Hierarchical chemosensory regulation of male-male social interactions in Drosophila. Nat Neurosci 14, 757-762.

Wang, L., Han, X., Mehren, J., Hiroi, M., Billeter, J.C., Miyamoto, T., Amrein, H., Levine, J.D., and Anderson, D.J. (2011b). Hierarchical chemosensory regulation of male-male 
social interactions in Drosophila. Nat Neurosci 14, 757-762.

Wang, Z., Singhvi, A., Kong, P., and Scott, K. (2004). Taste representations in the Drosophila brain. Cell 117, 981-991.

Wong, B.B.M., and Candolin, U. (2005). How is female mate choice affected by male competition? Biological Reviews 80, 559-571.

Wu, Q., Wen, T., Lee, G., Park, J.H., Cai, H.N., and Shen, P. (2003). Developmental control of foraging and social behavior by the Drosophila neuropeptide Y-like system. Neuron 39, 147-161.

Xu, K., Zheng, X., and Sehgal, A. (2008). Regulation of feeding and metabolism by neuronal and peripheral clocks in Drosophila. Cell metabolism 8, 289-300.

Xue, L., and Noll, M. (2000). Drosophila female sexual behavior induced by sterile males showing copulation complementation. Proc Natl Acad Sci U S A 97, 3272-3275.

Yamamoto, D. (2007). Genetics of sexual differentiation and sexually dimorphic behaviors. In Advances in genetics, (Amsterdam ; Boston: Elsevier/AP,), pp. 1 online resource (xii, 274 p., 278 p. of col. plates).

Yang, C.H., Rumpf, S., Xiang, Y., Gordon, M.D., Song, W., Jan, L.Y., and Jan, Y.N. (2009). Control of the postmating behavioral switch in Drosophila females by internal sensory neurons. Neuron 61, 519-526.

Yapici, N., Kim, Y.J., Ribeiro, C., and Dickson, B.J. (2008). A receptor that mediates the post-mating switch in Drosophila reproductive behaviour. Nature 451, 33-37.

Yu, J.Y., Kanai, M.I., Demir, E., Jefferis, G.S., and Dickson, B.J. (2010a). Cellular organization of the neural circuit that drives Drosophila courtship behavior. Current biology : CB 20, 1602-1614.

Yu, J.Y., Kanai, M.I., Demir, E., Jefferis, G.S., and Dickson, B.J. (2010b). Cellular organization of the neural circuit that drives Drosophila courtship behavior. Curr Biol 20, 1602-1614.

Yuan, Q., Song, Y., Yang, C.H., Jan, L.Y., and Jan, Y.N. (2014). Female contact modulates male aggression via a sexually dimorphic GABAergic circuit in Drosophila. Nat Neurosci 17, 81-88.

Zarkower, D. (2001). Establishing sexual dimorphism: conservation amidst diversity? Nat Rev Genet 2, 175-185.

Zhang, S.X., Rogulja, D., and Crickmore, M.A. (2016). Dopaminergic Circuitry Underlying Mating Drive. Neuron.

Zheng, X., and Sehgal, A. (2008). Probing the relative importance of molecular oscillations in the circadian clock. Genetics 178, 1147-1155.

Zheng, X., Yang, Z., Yue, Z., Alvarez, J.D., and Sehgal, A. (2007). FOXO and insulin signaling regulate sensitivity of the circadian clock to oxidative stress. Proc Natl Acad Sci U S A 104, 15899-15904. 
bioRxiv preprint doi: https://doi.org/10.1101/088724; this version posted November 23, 2016. The copyright holder for this preprint (which was

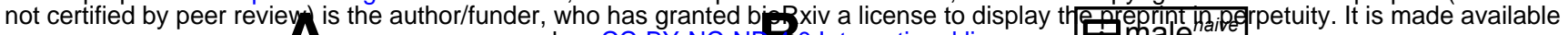
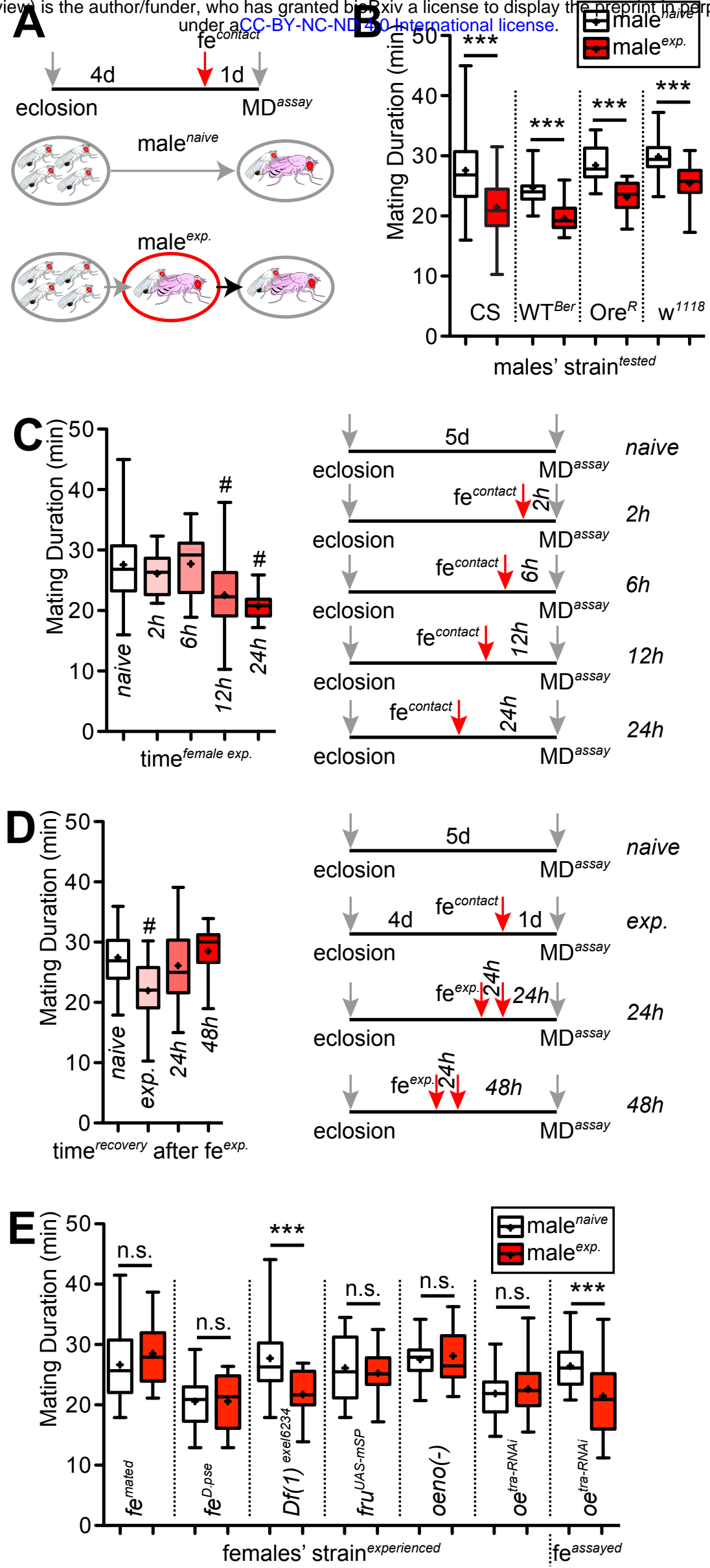

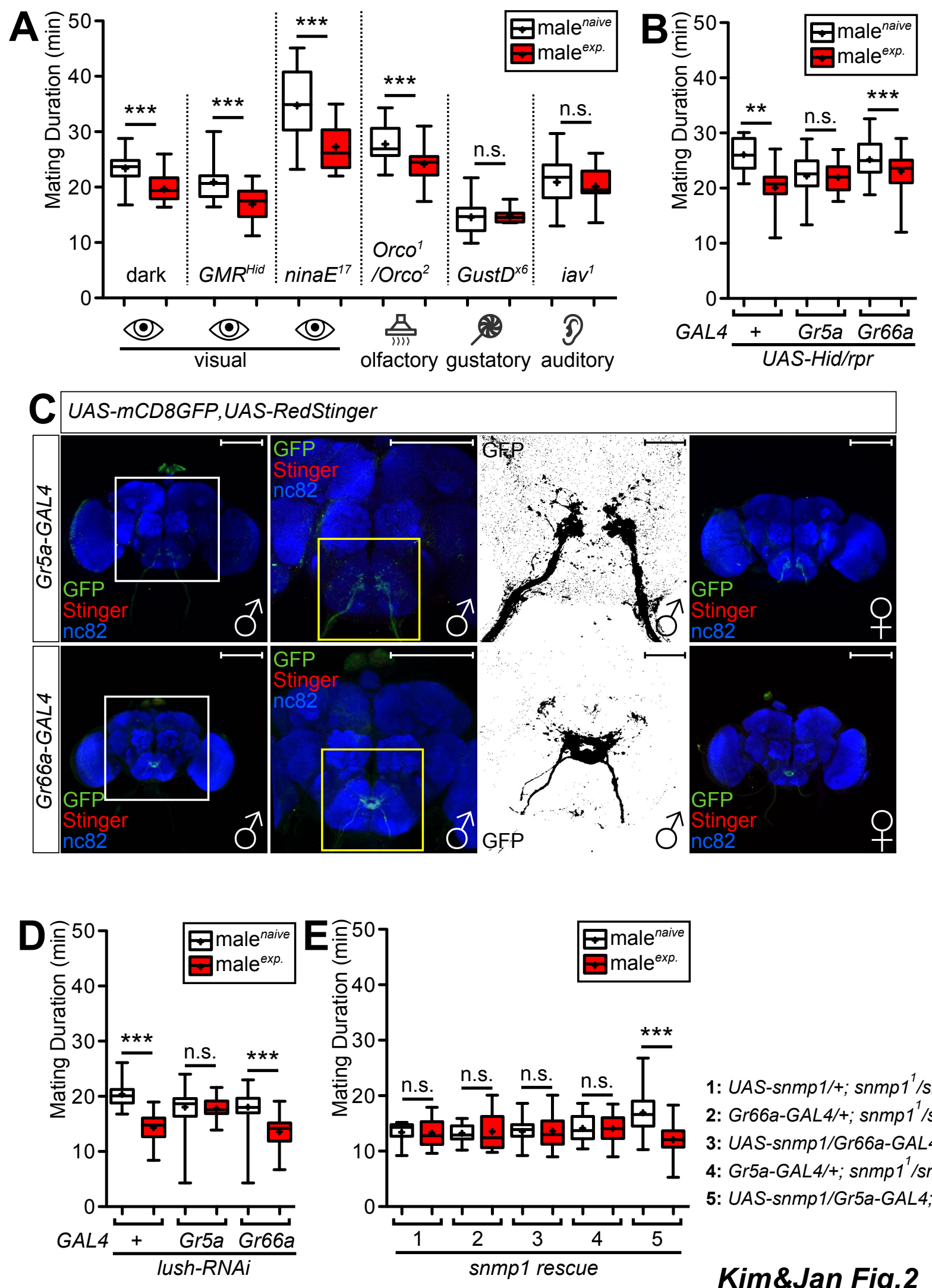

1: UAS-snmp $1 /+$; snmp $1^{1} /$ snmp $^{2}$

2: Gr66a-GAL4/+; snmp $1^{1} /$ snmp $^{2}$

3: UAS-snmp1/Gr66a-GAL4; snmp $1^{1} /$ snmp $^{2}$

4: Gr5a-GAL4/+; snmp $1^{1} /$ snmp $^{2}$

5: UAS-snmp $1 /$ Gr5a-GAL4; snmp $1 /$ snmp $1^{2}$

Kim\&Jan Fig. 2 


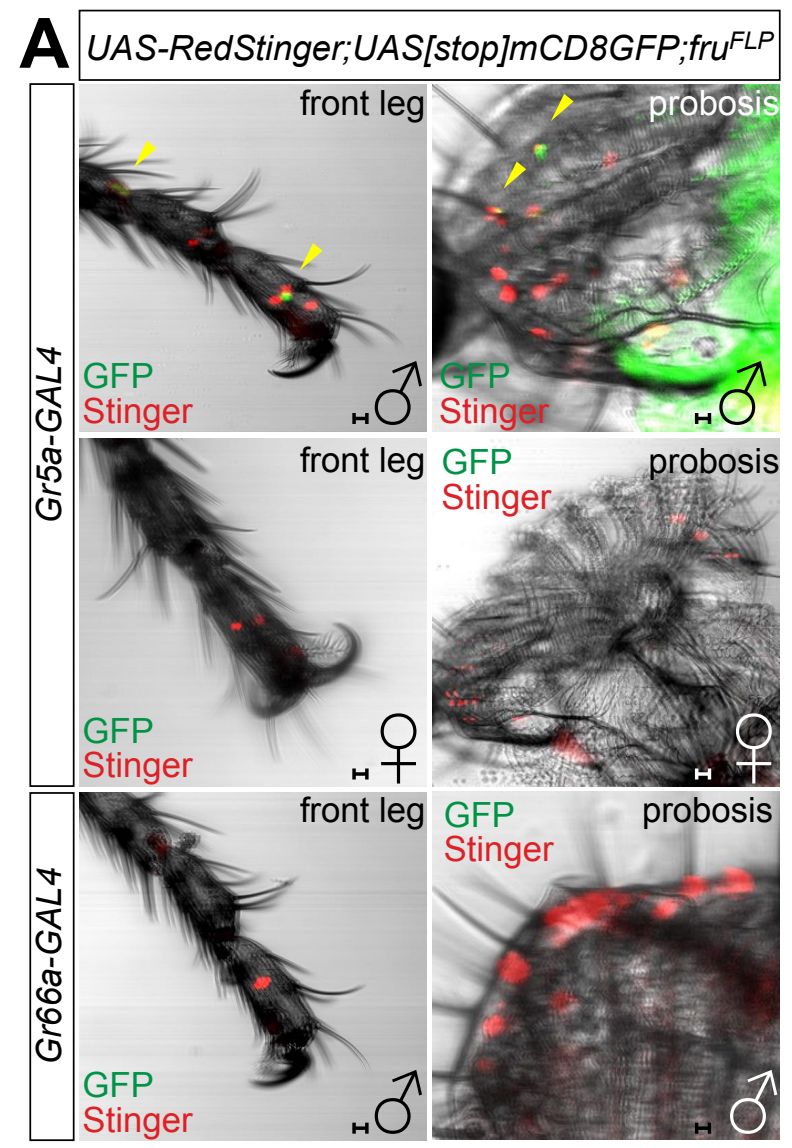

\section{B UAS[stop]mCD8GFP; fruFLP}

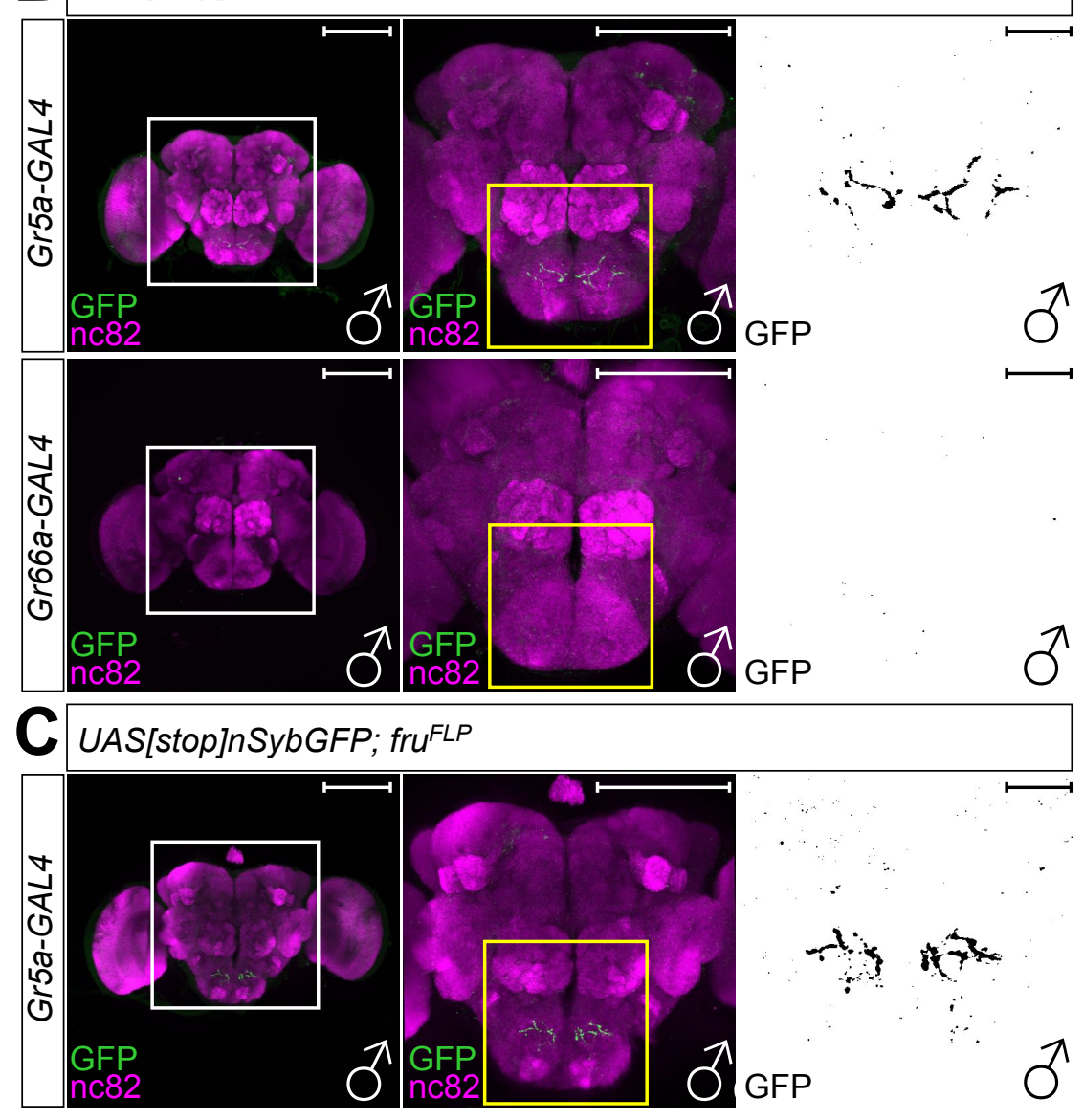

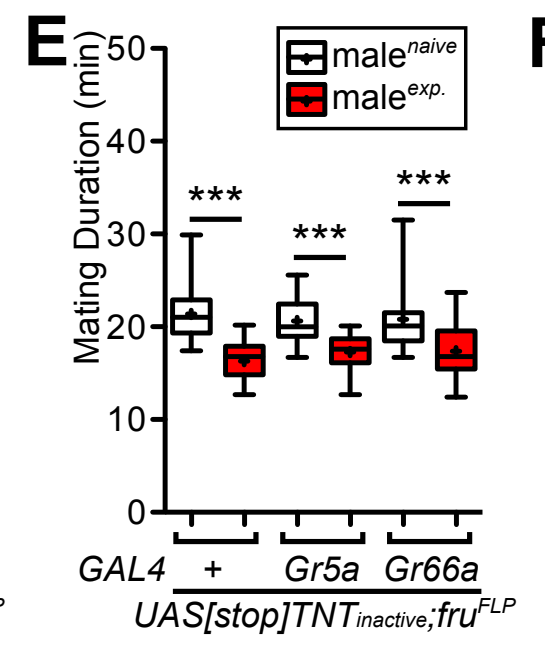

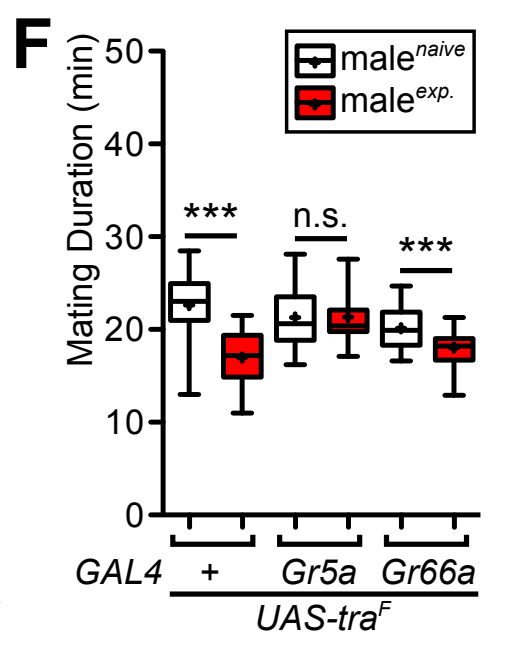



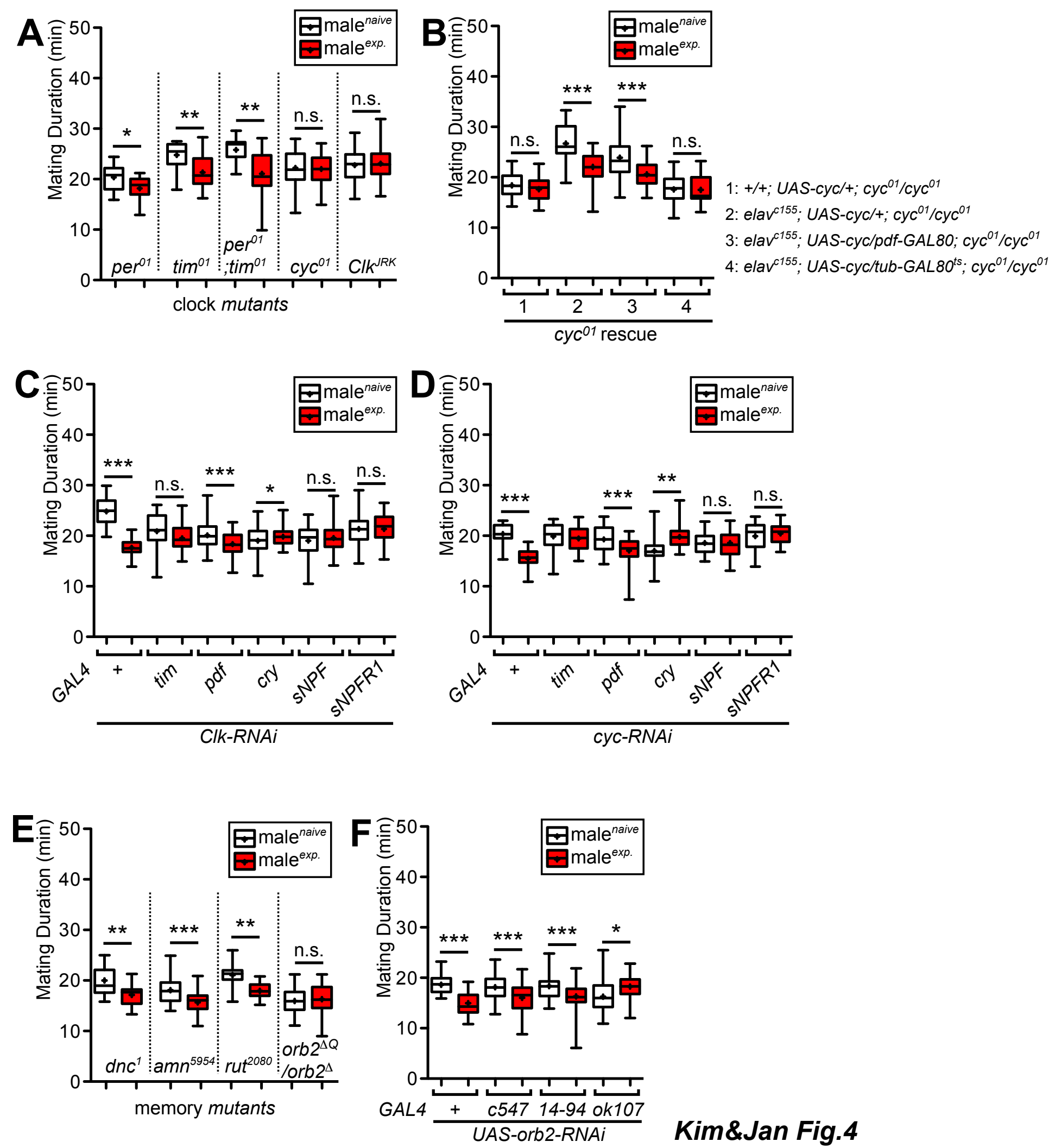

Kim\&Jan Fig.4 

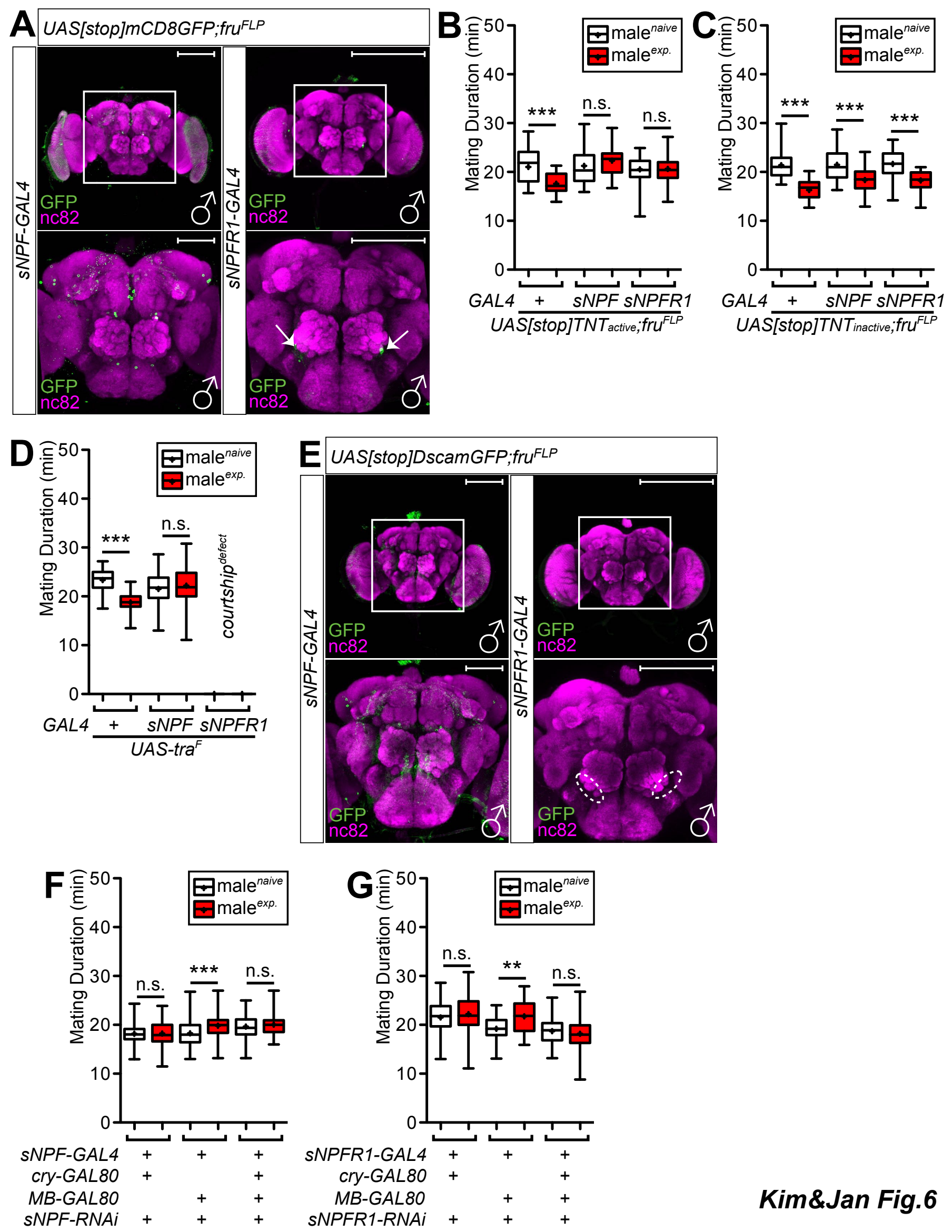

Kim\&Jan Fig. 6 

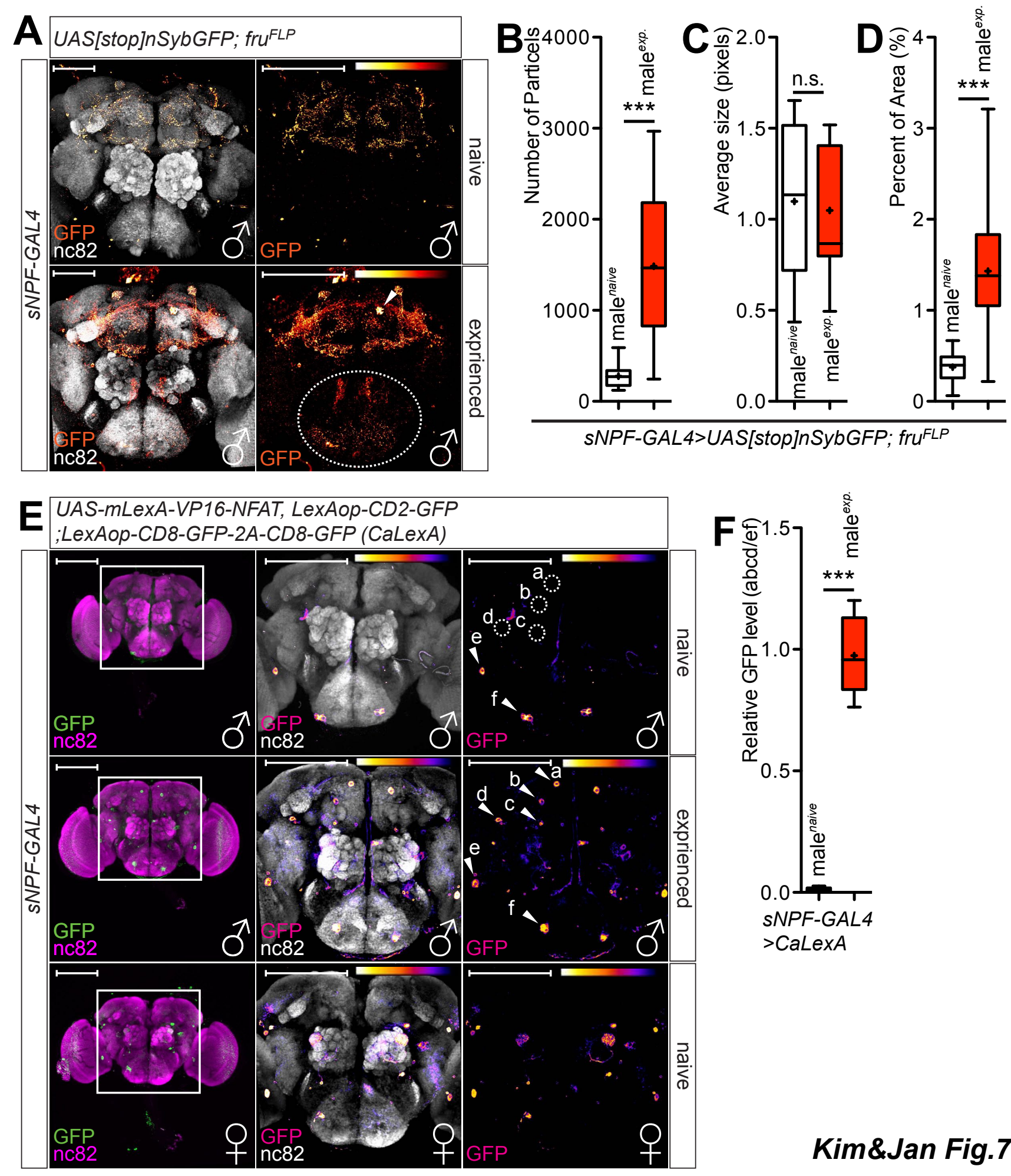

Kim\&Jan Fig.7 
A

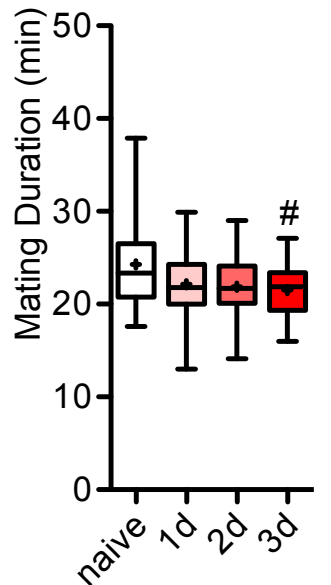

socially-isolated
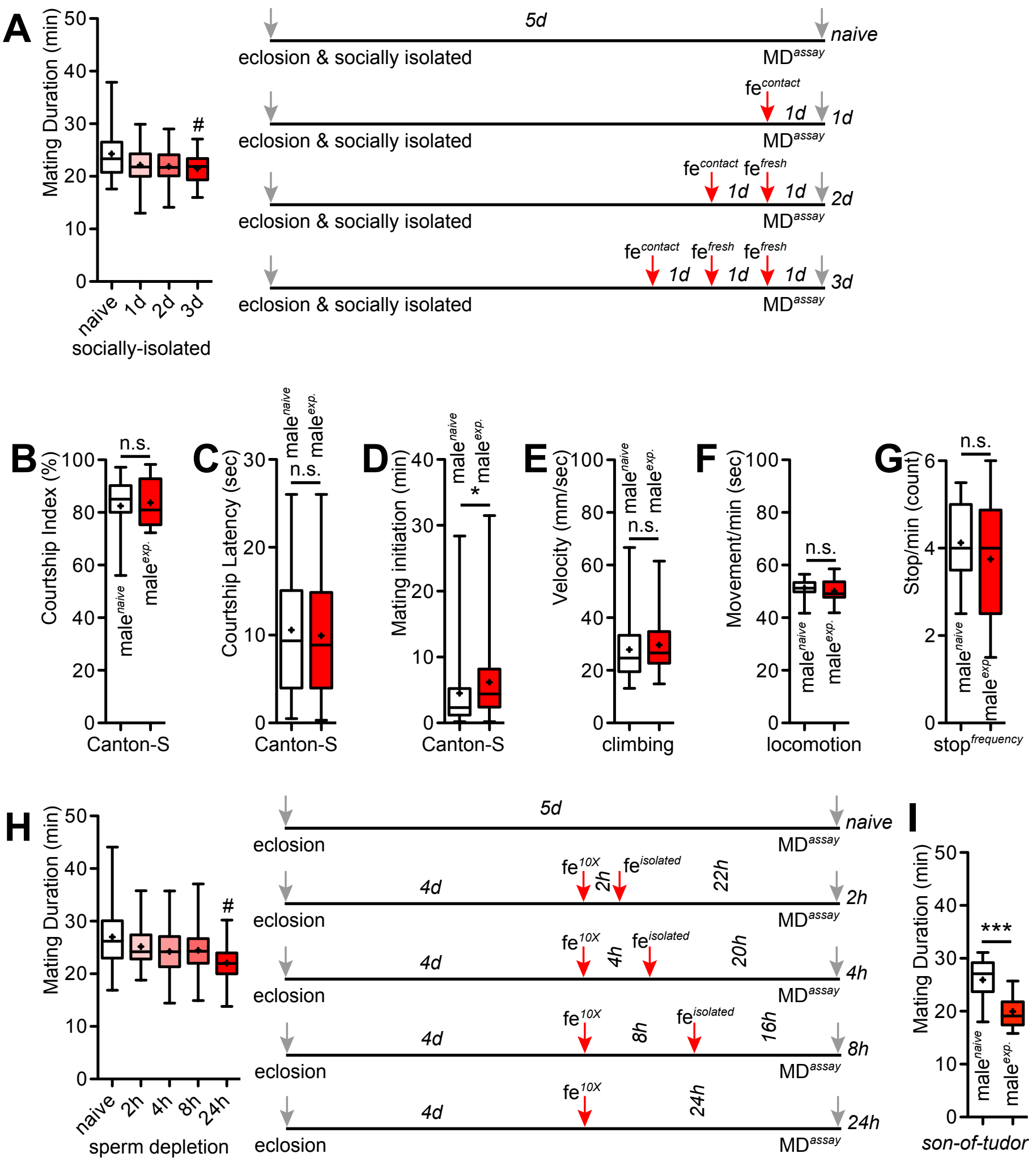

Kim\&Jan Supple.Fig.1 

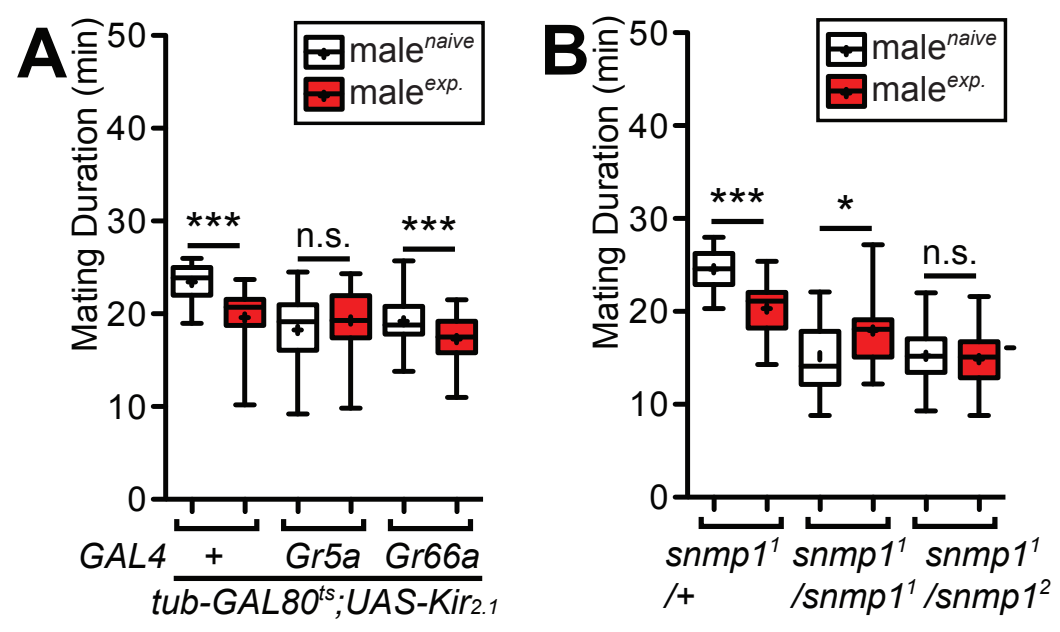

Kim\&Jan Supple.Fig. 2 


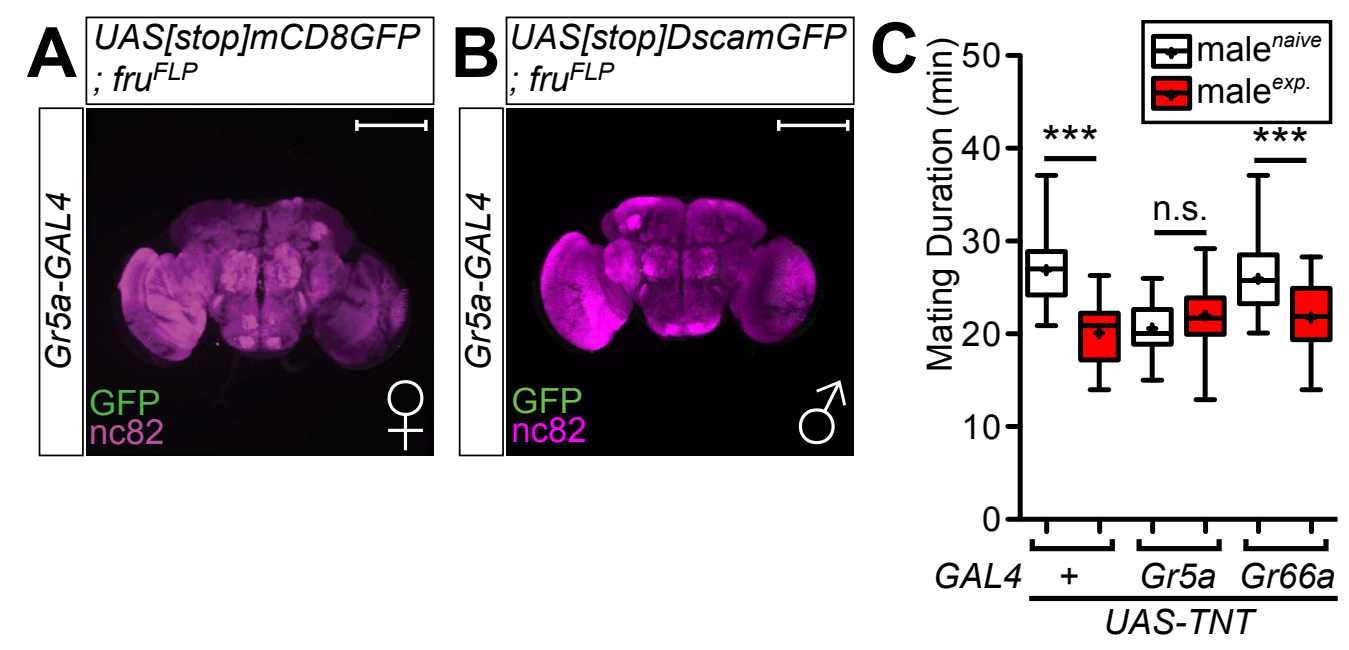



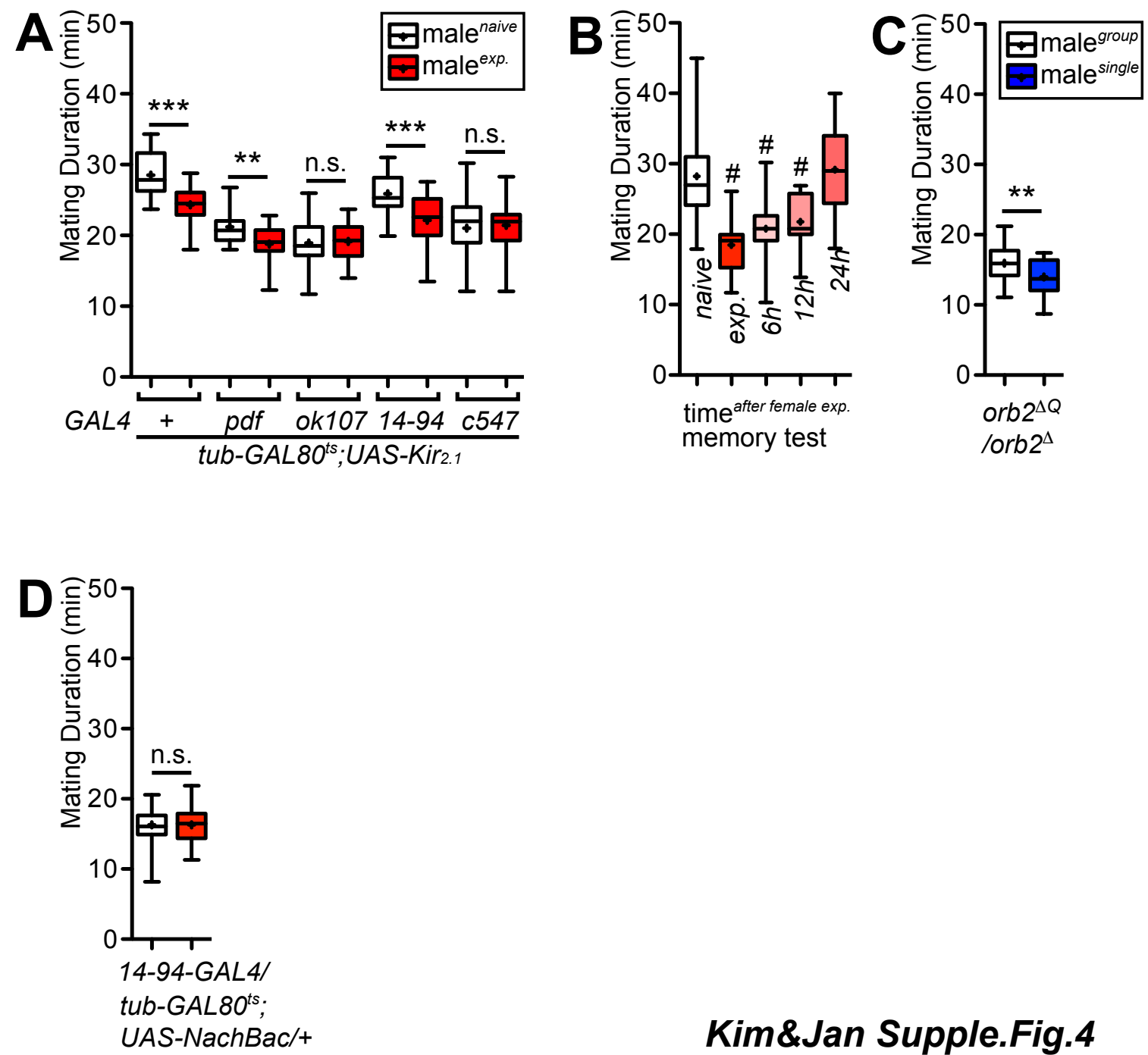

Kim\&Jan Supple.Fig.4 

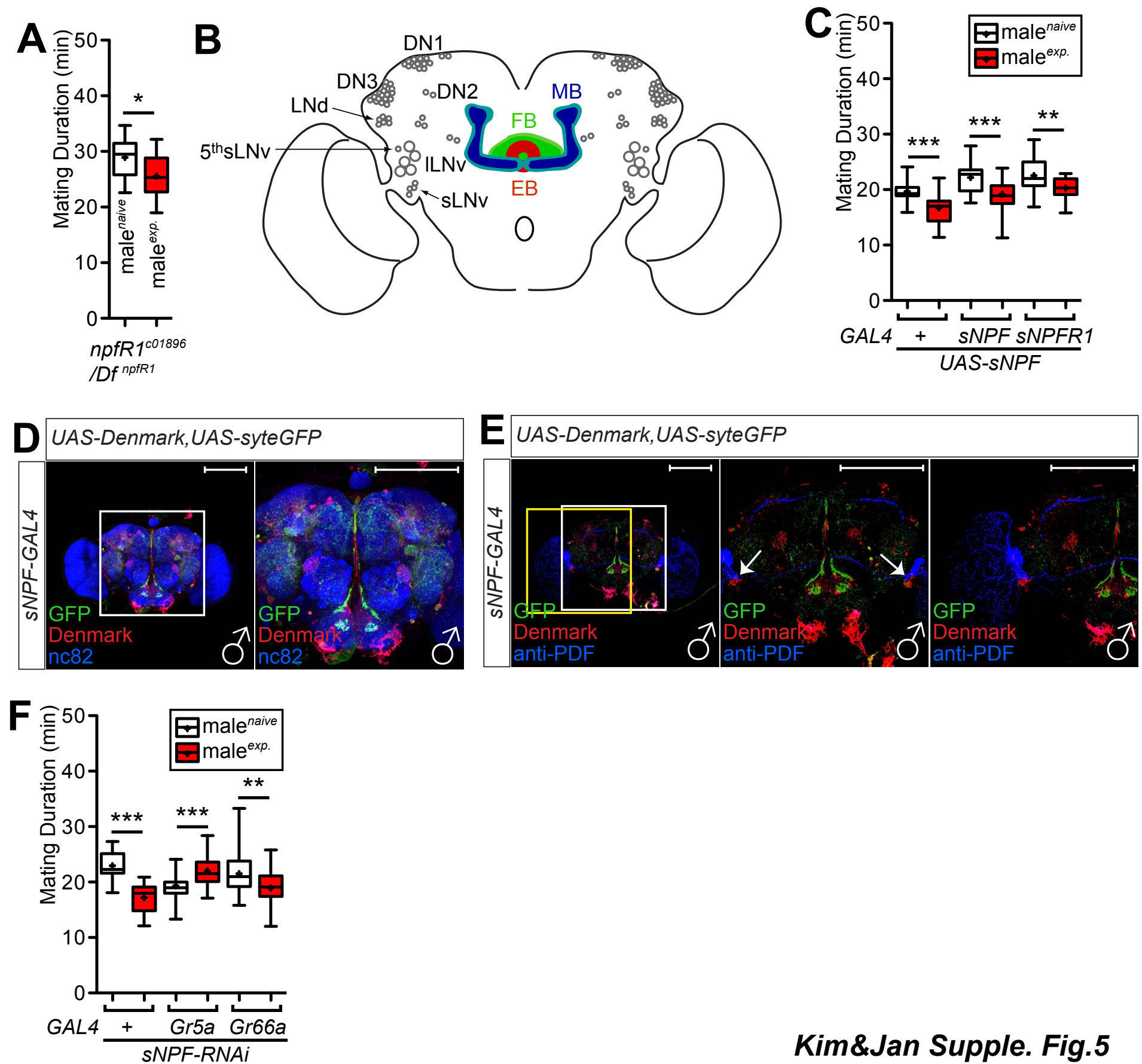

Kim\&Jan Supple. Fig.5 
bioRxiv preprint doi: https://doi.org/10.1101/088724; this version posted November 23, 2016. The copyright holder for this preprint (which was not certified by peer review) is the author/funder, who has granted bioRxiv a license to display the preprint in perpetuity. It is made available under aCC-BY-NC-ND 4.0 International license.
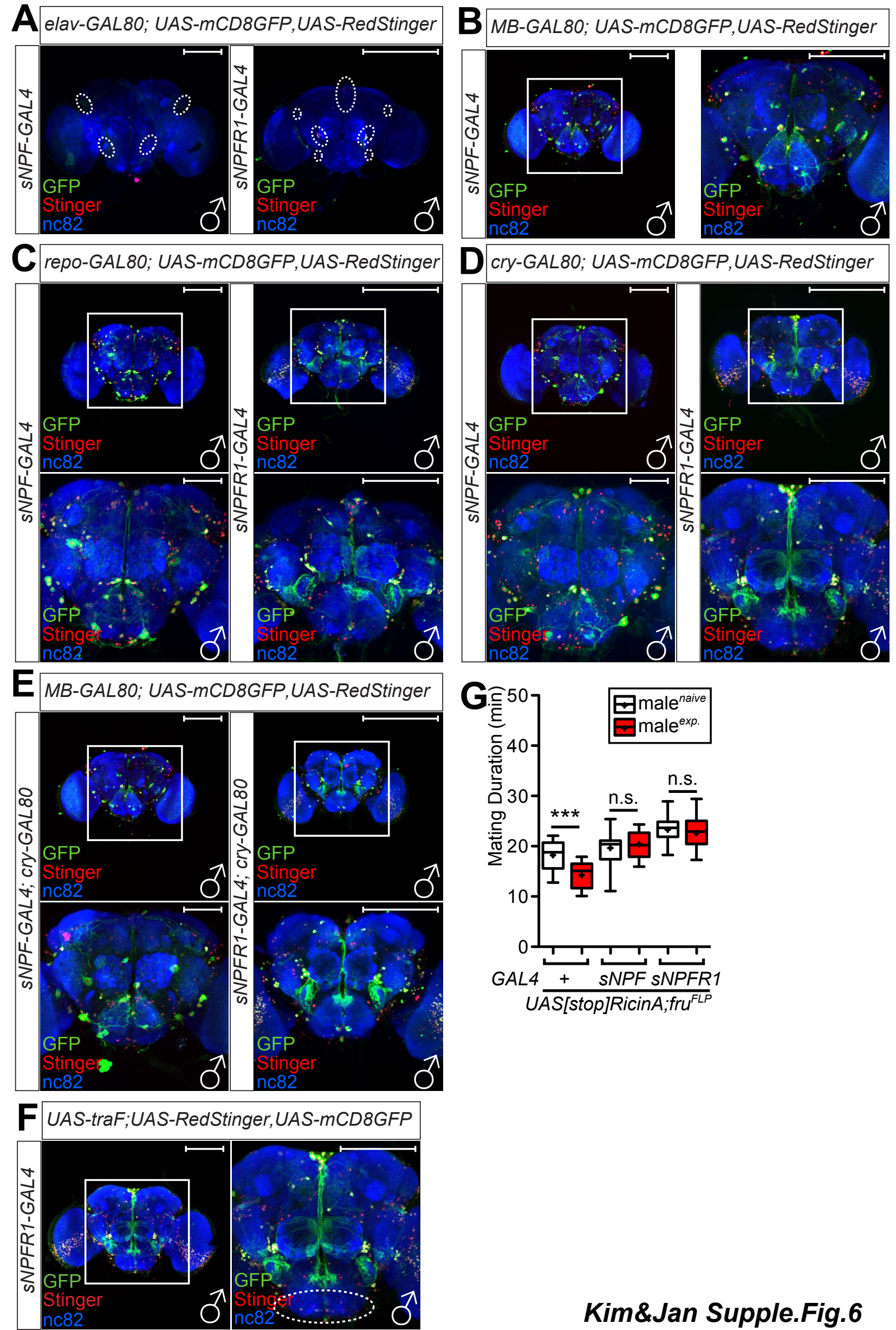


\begin{tabular}{ccc}
\hline & LMD $^{\text {a }}$ & SMD \\
\hline Sensory input & vision & taste, touch \\
Clock components & PER/TIM & CLK/CYC \\
Neuropeptide & PDF/NPF & sNPF \\
Memory circuit $($ gene $)$ & EB $($ rut, amn) & EB \& MB (orb2) \\
Sexual dimorphic cells & 2 LNd clock neurons & $\sim 4$ Gr5a taste neurons \\
\hline
\end{tabular}

Kim\&Jan Supple.Table.1 